\title{
Identification of novel genomic regions associated with nine mineral elements in Chinese winter wheat grain
}

Wei Wang ${ }^{1 \dagger}$, Hong Guo ${ }^{1 \dagger}$, Chongning Wu ${ }^{1}$, Hui Yu ${ }^{1}$, Xiaokang $\mathrm{Li}^{2}$, Guangfeng Chen ${ }^{3}$, Jichun Tian ${ }^{1}$ and Zhiying Deng ${ }^{1^{*}}$

\begin{abstract}
Background: Mineral elements are important for maintaining good human health besides heavy metals. Mining genes that control mineral elements are paramount for improving their accumulation in the wheat grain. Although previous studies have reported some loci for beneficial trace elements, they have mainly focused on Zn and Fe content. However, little information is available regarding the genetic loci differences in dissecting synchronous accumulation of multiple mineral elements in wheat grains, including beneficial and heavy elements. Therefore, a genome-wide association study (GWAS) was conducted on 205 wheat accessions with 24,355 single nucleotide polymorphisms (SNPs) to identify important loci and candidate genes for controlling $\mathrm{Ca}$, Fe, Zn, $\mathrm{Se}, \mathrm{Cu}, \mathrm{Mn}, \mathrm{Cd}$, As, and Pb accumulation in wheat grains.
\end{abstract}

Results: A total of 101 marker-trait associations (MTAs) $\left(P<10^{-5}\right)$ loci affecting the content of nine mineral elements was identified on chromosomes 1B, 1D, 2A, 2B, 3A, 3B, 3D, 4A, 4B, 5A, 5B, 5D, 6B, 7A, 7B, and 7D. Among these, 17 major MTAs loci for the nine mineral elements were located, and four MTAs loci $\left(P<10^{-5}\right)$ were found on chromosomes 1B, 6B, 7B, and 7D. Eight multi-effect MTAs loci were detected that are responsible for the control of more than one trait, mainly distributed on chromosomes 3B, 7B, and 5A. Furthermore, sixteen candidate genes controlling $\mathrm{Ca}$, Fe, $\mathrm{Zn}, \mathrm{Se}, \mathrm{Cd}$, and $\mathrm{Pb}$ were predicted, whose functions were primarily related to ion binding, including metals, $\mathrm{Fe}, \mathrm{Ca}, \mathrm{Cu}$, Mg, and Zn, ATP binding, ATPase activity, DNA binding, RNA binding, and protein kinase activity.

Conclusions: Our study indicated the existence of gene interactions among mineral elements based on multi-effect MTAs loci and candidate genes. Meanwhile this study provided new insights into the genetic control of mineral element concentrations, and the important loci and genes identified may contribute to the rapid development of beneficial mineral elements and a reduced content of harmful heavy metals in wheat grain.

Keywords: Wheat grain, Nine mineral elements, Genome wide association study, Candidate gene

*Correspondence: deng868@163.com

tWei Wang and Hong Guo contributed equally to this work.

${ }^{1}$ State Key Laboratory of Crop Biology, Key Laboratory of Crop Biology of Shandong Province, Group of Wheat Quality and Molecular Breeding, College of Agronomy, Shandong Agricultural University, Tai'an, Shandong 271000, P.R. China

Full list of author information is available at the end of the article

\section{Background}

Wheat is one of the most important crops grown globally, and as a staple food, it provides approximately $20 \%$ of the calories and $40 \%$ of the protein consumed worldwide. With the improvement in living standards, the nutritional quality of wheat grain has become increasingly important. There are many mineral elements in the wheat grain, that are important sources of trace elements in the human body. Although some important mineral 
elements can be found in wheat grains, such as iron $(\mathrm{Fe})$, zinc $(\mathrm{Zn})$, and selenium $(\mathrm{Se})$, their content is relatively low and their bioavailability is poor, which leads to the potential threat of mineral-element nutritional deficiencies in developing countries around the world [1].

More than one-third of the women and children in economically underdeveloped countries do not receive key trace elements, such as Fe, Zn, and iodine (I), in sufficient amounts to sustain a healthy condition. Specifically, more than two-thirds of the population in China suffers from Se deficiency [2]. Therefore, effectively improving the content of beneficial mineral elements in the wheat grain has become an issue of the highest priority in plant breeding programs.

In addition to some beneficial mineral elements, wheat also contains some heavy metals because of environmental pollution, such as $\mathrm{Cd}, \mathrm{Pb}$, and As, whose excessive intake may cause damage to human health, including cancer of the prostate, lungs, and testes, as well as kidney tubule damage $[3,4]$. Therefore, in recent years, the heavy metal content in food has become a focus of attention for the society as a whole. The essential and toxic nature of several dietary trace elements, including $\mathrm{Cr}$, $\mathrm{Co}, \mathrm{Cu}, \mathrm{Fe}, \mathrm{Mg}, \mathrm{Mn}, \mathrm{Se}$, and $\mathrm{Zn}$, have been thoroughly investigated [5-10].

Micronutrient deficiencies (especially $\mathrm{Zn}$ and Fe), are responsible for the effects of malnutrition on a very large proportion of the world population. In developing countries, most people rely on cereal grains as their staple food, and malnutrition has been detected among children because of micronutrient deficiencies, a phenomenon that has been described as the 'hidden hunger' $[1,11$, 12]. To solve malnutrition caused by the lack of beneficial mineral elements in food, biofortification of wheat grain has become a routine in the food industry, especially for $\mathrm{Zn}, \mathrm{Fe}$, and Se. Since 2003, scientists from various international agricultural research institutes have begun to implement the Harvest Plus project to solve this problem by cultivating mineral-rich food crops. Generally, there are two methods used to improve micronutrient content of wheat grain, which involves agronomic practices and genetic improvement. However, agronomic practices are reputedly neither economical nor environmentally friendly [13].

Alternatively, the genetic improvement for micronutrient content such as $\mathrm{Zn}, \mathrm{Fe}$, and Se in wheat grain is an important and effective approach. In previous studies, some important genes/loci for micronutrients including, $\mathrm{Zn}, \mathrm{Fe}$, and $\mathrm{Se}$, were found using quantitative trait loci (QTL) mapping and genome-wide association study (GWAS) methods. Thus, using three different sets of recombinant inbred lines (RILs) [14, 15] and QTL mapping, two major QTLs for Zn content on chromosome
7B (QGZn.cimmyt-7B_1P2 and QGZn.cimmyt-7B_1P1) and one major QTL for Fe content on chromosome 4A (QGFe.cimmyt-4A_P2) were identified, and pleiotropic QTLs were also found on chromosome 3B. Additionally, through the meta-QTL (MQTL) method, some important MQTLs for $\mathrm{Zn}$ and Fe content were found on chromosomes 2D, 5A, 5B, 6A, and 7A [13]. However, there were only three studies reported for the QTLs of Se content on 21 chromosomes [16-18].

Using association panels, including common wheat (Triticum aestivum L.), synthetic hexaploid wheat, harvest plus association panel, European wheat varieties, and spring wheat, a total of 442 marker-trait associations (MTAs) for Zn content and 287 MATs for Fe content were identified [19-27]. Of these, two highly significant MATs for $\mathrm{Zn}$ content were found on chromosomes $5 \mathrm{~A}$ and $3 \mathrm{~B}$, and six candidate genes on $3 \mathrm{BS}$ were predicted to belong to the mitogen-activated protein kinase family, which is involved in protein kinase activity, protein phosphorylation, and protein transport [13]. This is related to $\mathrm{Zn}$ uptake and transport. On chromosome 5AL, four candidate genes were found in the bZip family and FAR1 protein, which are related to Zn biofortification [13]. Furthermore, the $G p c-b 1$ gene cloned from wild wheat has been shown to increase protein, $\mathrm{Zn}$, and $\mathrm{Fe}$ content in wheat grain [28].

Although previous studies have reported some loci for beneficial trace elements, they have mainly focused on $\mathrm{Zn}$ and Fe content. However, little information is available regarding the genetic loci differences in dissecting synchronous accumulation of multiple mineral elements in wheat grains, including beneficial and heavy elements. Therefore, this study used GWAS to dissect the accumulation of six beneficial elements and three harmful heavy elements using 24,355 single nucleotide polymorphisms (SNPs) genotyped from the $90 \mathrm{~K}$ Illumina iSelect array in a population of diverse winter wheat varieties. The objective of this study was to identify SNPs markers and candidate genes for loci associated with these traits, and improve the micronutrient content, and reduce the threat of heavy metals in the wheat grain through molecular breeding. Our results will provide the theoretical basis for improving grain micronutrient content without increasing harmful mineral elements through molecular marker-assisted selection.

\section{Materials and methods \\ Plant material}

The association mapping panel of 205 wheat genotypes for GWAS comprised 77 released cultivars, 55 landraces, including two lines from Mexico and France, and 73 breeding lines from 10 provinces representing the major winter wheat-production regions in China [29]. About 
thirty seeds per each of these materials were originally acquired from National Germplasm Bank, Shandong Germplasm Bank, Academy of Agricultural Sciences of different province, and wheat breeders. And then they were multiply reproduced in our research field by our Research Group of Wheat Quality breeding from Shandong Agricultural University, Shandong Province, China. The details were seen in previous published paper [29].

\section{Growth conditions}

The seeds used for the association mapping panel were planted in the 2014, 2015, 2016, and 2017 growing seasons in experimental fields at two locations: Shandong Agricultural University, Tai'an (TA, $36^{\circ} 57$ N 116 $36 \mathrm{E}$ ) and the Dezhou Institute of Agricultural Sciences, Dezhou (DZ, $\left.37^{\circ} 45 \mathrm{~N} 116^{\circ} 29 \mathrm{E}\right)$. E1, E2, E3, and E4 represented the Dezhou location in 2014 (2014 DZ), Tai'an location in 2015 (2015 TA), Tai'an location in 2016 (2016 TA), and Tai'an location in 2017 (2017 TA), respectively. All experiments were laid in a completely randomised block design with two replicates in each environment. All lines were grown in $1.3 \mathrm{~m}$ plots with three rows spaced $25 \mathrm{~cm}$ apart, and 40 seeds evenly broadcast in each row. All recommended local crop management practices were followed during all the growing seasons, and no damage attributed to lodging, disease, or pests was observed.

The soil conditions at different locations are shown in Table S1. There were no significant differences in different mineral elements between the two locations. This indicated that the soil conditions at the two locations appeared to be the same.

\section{Phenotypic trait evaluation Whole flour milling}

Grain samples were washed three times with distilled water to remove any attached particles and then ovendried at $80{ }^{\circ} \mathrm{C}$. Dried grain samples $(50 \mathrm{~g})$ were milled using a whole flour experimental mill (Perten 3100 type mill, Perten Co.,Stockholm, Sweden).

\section{Determination of metal element content in whole flour} Whole flour $(0.2 \mathrm{~g})$ samples were introduced into digestion tubes for digestion with $6 \mathrm{~mL}$ of nitric acid $\left(\mathrm{HNO}_{3}\right)$ in a microwave digester. The digested solutions were filtered through a $0.45 \mu \mathrm{m}$ water-based microporous membranes after dilution to a constant volume of $50 \mathrm{~mL}$ with deionised water. Subsequently, the concentrations of different metal elements including, $\mathrm{Ca}, \mathrm{Mn}, \mathrm{Fe}, \mathrm{Cu}, \mathrm{Zn}$, $\mathrm{Se}, \mathrm{As}, \mathrm{Cd}$, and $\mathrm{Pb}$, were determined using inductively coupled plasma-atomic emission spectrometry (ICPMS, Thermo Fisher, iCAP Qc). The standard curves for the different metal elements are shown in Fig. S1, and the correlation linear valuer [2]. was between 0.9977 and 0.9999 . The recovery rates of all the elements ranged between 80 and $120 \%$.

\section{Statistical analysis}

An analysis of variance (ANOVA) and correlations among phenotypic traits were conducted using the PROC GLM procedure of SAS 8.0 (SAS Institute Inc., Cary, NC, USA) and the statistical software SPSS version 17.0 (SPSS Inc., Chicago, IL, USA), respectively.

\section{Genome-wide association analysis}

SNP markers, genotyping, and the population structure of the samples have been previously reported [30]. Based on this information, significant MTAs were identified using a mixed linear model (MLM) in TASSEL3.0. The P-value was used to determine whether a QTL was associated with a marker. The $R$ [2] value was used to evaluate the magnitude of the MTA effects. The genome-wide significance threshold $\left(\mathrm{P} \leq 10^{-4}\right)$ was determined. SNPs with a P-value $\leq 10^{-4}$ were considered to be significantly associated with phenotypic traits. When an MTA locus was detected in two or more environments, it was considered a site-stable association [29].

\section{Candidate genes prediction for important MTAs loci associated with mineral elements}

To identify the position of important MTAs loci and possible candidate genes on a physical map, significant markers detected in this study were used to identify putative candidate genes. A BLAST (Basic Local Alignment Search Tool) search was performed using the International Wheat Genome Sequencing Consortium database (IWGSC; http://www.wheatgenome.org/, 20 ${ }^{\text {th }}$ January 2021) with the sequence of the significant SNP markers identified by GWAS. When an SNP marker sequence from the IWGSC was $100 \%$ identical to any wheat contig, the sequence was extended by $2 \mathrm{Mb}$ for each marker using the IWGSC BLAST results. The extended sequence was used to run a BLAST search at the National Center for Biotechnology Information (NCBI) database (http:// www.ncbi.nlm.nih.gov, $20^{\text {th }}$ January 2021) and Ensembl Plants (http://plants.ensembl.org/Triticum_aestivum/ Tools/Blast, $20^{\text {th }}$ January 2021) to confirm possible candidate genes and functions.

\section{Results}

Phenotypic variation and correlation analysis for nine mineral elements

Extensive phenotypic variations in $\mathrm{Ca}, \mathrm{Mn}, \mathrm{Fe}, \mathrm{Cu}, \mathrm{Zn}$, $\mathrm{Se}, \mathrm{As}, \mathrm{Cd}$, and $\mathrm{Pb}$ were observed among the 205 winter wheat accessions across the four environments (i.e. 3 years and two locations, Table 1). Continuous distributions in the population were observed for nine mineral 
Table 1 Phenotypic variation of beneficial and harmful mineral elements in wheat grain

\begin{tabular}{|c|c|c|c|c|c|c|c|}
\hline Trait & Environment & Minimum $(\mu \mathrm{g} / \mathrm{mL})$ & Maximum $(\mu \mathrm{g} / \mathrm{mL})$ & Mean $(\mu \mathrm{g} / \mathrm{mL})$ & Standard deviation & Kurtosis & Skewness \\
\hline \multirow[t]{4}{*}{$\mathrm{Ca}$} & E1 & 1305.3412 & 3494.3349 & $2057.7033 \mathrm{aA}$ & 131.1936 & 0.3429 & 0.4879 \\
\hline & E2 & 943.2681 & 3851.0267 & $1671.1812 \mathrm{cC}$ & 135.3059 & 4.2332 & 1.4296 \\
\hline & E3 & 1041.3682 & 3571.2884 & $1904.1424 b B$ & 142.1176 & 0.9116 & 0.8325 \\
\hline & E4 & 745.5555 & 4337.0893 & $1635.2395 c C$ & 155.1693 & 8.3606 & 2.1744 \\
\hline \multirow[t]{4}{*}{$\mathrm{Mn}$} & E1 & 72.3929 & 177.8321 & 114.308 9abAB & 7.1707 & 0.4113 & 0.7809 \\
\hline & E2 & 64.6798 & 233.9433 & $110.9381 \mathrm{bB}$ & 7.1124 & 7.0999 & 1.6444 \\
\hline & E3 & 71.5679 & 170.7556 & $110.7514 \mathrm{bB}$ & 6.0726 & 0.3201 & 0.4435 \\
\hline & E4 & 78.8852 & 177.4753 & $117.4769 \mathrm{aA}$ & 6.1596 & 0.1513 & 0.6035 \\
\hline \multirow[t]{4}{*}{$\mathrm{Fe}$} & E1 & 49.4330 & 275.9624 & $122.3629 \mathrm{cC}$ & 14.8955 & -0.3778 & 0.5288 \\
\hline & E2 & 44.1914 & 1267.6369 & $160.4930 \mathrm{bB}$ & 55.5250 & 15.7235 & 3.6502 \\
\hline & E3 & 60.0906 & 1226.4099 & 195.083 0aA & 72.5161 & 9.5907 & 3.0478 \\
\hline & E4 & 41.7784 & 810.9322 & $137.2284 \mathrm{bcBC}$ & 34.8879 & 19.8211 & 4.0365 \\
\hline \multirow[t]{4}{*}{$\mathrm{Cu}$} & E1 & 8.7895 & 20.8090 & $14.5411 \mathrm{aA}$ & 0.8543 & -0.5372 & 0.2307 \\
\hline & E2 & 6.5942 & 38.2897 & $11.1470 \mathrm{dD}$ & 0.9059 & 49.9301 & 5.1855 \\
\hline & E3 & 6.4520 & 19.3701 & $12.1055 \mathrm{cC}$ & 0.7183 & 0.9057 & 0.4956 \\
\hline & E4 & 8.1342 & 31.3270 & 13.098 8bB & 0.8790 & 10.9452 & 1.8871 \\
\hline \multirow[t]{4}{*}{ Zn } & E1 & 58.8303 & 391.2403 & $104.6091 \mathrm{aA}$ & 9.3453 & 53.8106 & 5.5144 \\
\hline & E2 & 40.1045 & 166.7603 & $81.535 \mathrm{OdC}$ & 7.0310 & 3.0504 & 1.4489 \\
\hline & E3 & 52.5687 & 156.9712 & $91.9332 \mathrm{cB}$ & 6.3487 & 1.1226 & 0.7969 \\
\hline & E4 & 61.4559 & 275.9468 & $99.2993 \mathrm{bA}$ & 8.6705 & 19.4522 & 3.6209 \\
\hline \multirow[t]{4}{*}{$\mathrm{Se}$} & E1 & 0 & 0.0045 & $0.0016 \mathrm{aA}$ & 0.0009 & 0.5010 & 0.0690 \\
\hline & E2 & 0 & 0.0045 & $0.0007 c B$ & 0.0009 & 1.4260 & 1.7290 \\
\hline & E3 & 0 & 0.0051 & $0.0009 \mathrm{bB}$ & 0.0009 & 1.2990 & 1.9680 \\
\hline & E4 & 0 & 0.0036 & $0.0007 c B$ & 0.0008 & 1.1650 & 0.7670 \\
\hline \multirow[t]{4}{*}{ As } & E1 & 0 & 0.0022 & $0.0001 \mathrm{cC}$ & 0.0003 & 3.9590 & 16.5060 \\
\hline & E2 & 0 & 0.0102 & $0.0008 \mathrm{bB}$ & 0.0015 & 3.3970 & 15.0580 \\
\hline & E3 & 0 & 0.0045 & $0.0002 \mathrm{cC}$ & 0.0005 & 5.5030 & 37.0330 \\
\hline & E4 & 0 & 0.0082 & $0.0019 \mathrm{aA}$ & 0.0018 & 1.1560 & 1.2480 \\
\hline \multirow[t]{4}{*}{$\mathrm{Cd}$} & E1 & 0 & 0.0179 & $0.0013 \mathrm{aA}$ & 0.0014 & 102.4070 & 8.8480 \\
\hline & E2 & 0 & 0.0203 & $0.0004 \mathrm{cC}$ & 0.0016 & 135.4680 & 10.8520 \\
\hline & E3 & 0 & 0.0144 & $0.0008 \mathrm{bB}$ & 0.0012 & 73.8880 & 7.3480 \\
\hline & E4 & 0 & 0.0161 & $0.0005 c B C$ & 0.0014 & 86.9770 & 8.5200 \\
\hline \multirow[t]{4}{*}{$\mathrm{Pb}$} & E1 & 0 & 0.2133 & - & - & - & - \\
\hline & E2 & 0 & 0.0988 & - & - & - & - \\
\hline & E3 & 0 & 0.2148 & - & - & - & - \\
\hline & E4 & 0 & 0.6926 & - & - & - & - \\
\hline
\end{tabular}

E1: 2014DZ; E2: 2015TA; E3: 2016TA; E4: 2017TA

elements, which showed typical quantitative traits, indicating that they were genetically controlled by multiple genes.

The ANOVA showed significant differences for the content of $\mathrm{Ca}, \mathrm{Mn}, \mathrm{Fe}, \mathrm{Cu}, \mathrm{Zn}, \mathrm{Se}$, and $\mathrm{Cd}(P<0.0001)$ among genotypes and environments, as well as $\mathrm{G} \times \mathrm{E}$ interactions (Table S2), which indicated that mineral element content was significantly affected by genotype, environment, and their interaction. Further, correlation analysis indicated that significant positive correlations were observed among $\mathrm{Ca}, \mathrm{Mn}, \mathrm{Fe}, \mathrm{Cu}$, and $\mathrm{Zn}$, but not $\mathrm{Se}$ (Table 2).

\section{Marker-trait associations (MTAs) of beneficial mineral elements}

A total of 64 MTAs $\left(P<10^{-4}\right)$ for Ca content in wheat grains were detected, mainly distributed on chromosomes $1 \mathrm{~A}, 2 \mathrm{~A}, 2 \mathrm{~B}, 3 \mathrm{~A}, 3 \mathrm{~B}, 5 \mathrm{~A}, 5 \mathrm{~B}, 5 \mathrm{D}, 6 \mathrm{~B}, 7 \mathrm{~A}$, and $7 \mathrm{~B}$ in the four experimental environments (Table S3). Some MTA clusters were found on chromosomes 1A, 2B, 3B, 
Table 2 Correlation analysis of beneficial mineral elements

\begin{tabular}{llllll}
\hline & $\mathbf{C a}$ & Mn & Fe & Cu & Zn \\
\hline $\mathrm{Ca}$ & 1 & & & & \\
$\mathrm{Mn}$ & $0.344^{* *}$ & 1 & 1 & & \\
$\mathrm{Fe}$ & $0.237^{* *}$ & $0.277^{* *}$ & $0.436^{* *}$ & 1 & 1 \\
$\mathrm{Cu}$ & $0.359^{* *}$ & $0.575^{* *}$ & $0.202^{* *}$ & $0.537^{* *}$ & -0.099 \\
$\mathrm{Zn}$ & $0.484^{* *}$ & $0.485^{* *}$ & -0.024 & 0.022 & 1 \\
$\mathrm{Se}$ & -0.085 & -0.020 & & & \\
\hline
\end{tabular}

** The correlation coefficient was very significant at $P<0.01$ level

${ }^{*}$ Correlation is significant at $P<0.01$

$5 \mathrm{~A}$, and $6 \mathrm{~B}$. Four MTAs were identified $\left(P<10^{-5}\right)$ on chromosomes $3 \mathrm{~B}$ and $5 \mathrm{~A}$ (Table 3 and Fig. S2), and the Kukri_c41797_393 locus on chromosome 5A contributed to the phenotypic variation with $10.97 \%$, as did the RFL_Contig2187_1025 locus.

For $\mathrm{Mn}$ content of the wheat grain, a total of 66 MTAs loci $\left(P<10^{-4}\right)$ were identified on chromosomes $1 \mathrm{~B}, 2 \mathrm{~A}, 2 \mathrm{~B}, 3 \mathrm{~A}, 4 \mathrm{~A}, 4 \mathrm{~B}, 5 \mathrm{~A}, 5 \mathrm{~B}$, and $7 \mathrm{~B}$ in three environments (Table S3). Three MTAs clusters were found on chromosomes 1B, 5A, and 7B. Of these, 14 MTAs loci at a genetic position of $75 \mathrm{cM}$ of chromosome $1 \mathrm{~B}$ were found at $P<10^{-5}$ levels in E2 (Table 3).

In turn, 77 MTAs loci $\left(P<10^{-4}\right)$ were identified to be associated with Fe content on 13 chromosomes (1A, 1D, $3 \mathrm{~A}, 4 \mathrm{~A}, 4 \mathrm{~B}, 4 \mathrm{D}, 5 \mathrm{~A}, 5 \mathrm{~B}, 6 \mathrm{~B}, 7 \mathrm{~A}, 7 \mathrm{~B}$ and $7 \mathrm{D})$ in the four experimental environments (Table S3). MTAs clusters were detected on chromosomes $5 \mathrm{~A}, 6 \mathrm{~B}, 7 \mathrm{~B}$, and 7D. Among them, 20 MTAs loci were found at the $P<10^{-5}$ level in E2, E3, and E4, explaining $8.07 \%$ to $16.23 \%$ of the phenotypic variation (Table 3). Ten MTAs loci were concentrated on the genetic position $26 \mathrm{cM}-27 \mathrm{cM}$ on chromosome 7D. Five MTAs loci explained more than $10 \%$ of the phenotypic variation on chromosomes $6 \mathrm{~B}$ and 7B. The Excalibur_c19455_3496 locus explained the maximum phenotypic variation observed (16.23\%) on chromosome 7B.

With respect to $\mathrm{Cu}, 96$ MTAs loci $\left(P<10^{-4}\right)$ controlling $\mathrm{Cu}$ content in wheat grain were detected on 15 chromosomes in the four experimental environments (Table S3). Some MTAs clusters were found on chromosomes $1 \mathrm{~A}, 1 \mathrm{~B}$, and $5 \mathrm{~A}$. However, at $P<10^{-5}$, there was only one locus, Excalibur_c29255_366, on chromosome 4 B (Table 3).

As for $\mathrm{Zn}$ content in wheat grain, there were 95 loci $\left(P<10^{-4}\right)$ identified on 17 chromosomes, except for chromosomes 2A, 2D, 6A, and 6D in the four experimental environments (Table S3). MTAs clusters were found mainly on $2 \mathrm{~B}, 3 \mathrm{D}, 5 \mathrm{~B}, 6 \mathrm{~B}$, and $7 \mathrm{~B} ; 16$ MTAs loci were found at the $P<10^{-5}$ level in E1, E3, and E4 (Table 3). These three explained more than $10 \%$ of the phenotypic variation on chromosomes $3 \mathrm{~B}$ and $4 \mathrm{~B}$. The BS00057451_51 locus exhibited the maximum (13.25\%) phenotypic variation observed.

With respect to Se, 57 MTAs loci $\left(P<10^{-4}\right)$ controlling Se content in wheat grain were identified on chromosomes $1 \mathrm{~A}, 2 \mathrm{~B}, 3 \mathrm{~A}, 3 \mathrm{D}, 4 \mathrm{~A}, 4 \mathrm{~B}, 5 \mathrm{~A}, 6 \mathrm{~B}, 7 \mathrm{~A}, 7 \mathrm{~B}$, and 7D in the four experimental environments (Table S4). Some MTAs clusters were found on chromosomes $5 \mathrm{~A}, 6 \mathrm{~B}$, and $7 \mathrm{~B}$, and six MATs loci were detected at the $P<10^{-5}$ level (Table 4), but only one major MAT with $10.06 \%$ phenotypic variation was detected on chromosome 3D.

\section{MTAs of heavy metal elements}

For the As content in wheat grain, 67 MTAs loci $\left(P<10^{-4}\right)$ were identified on 14 chromosomes $(1 \mathrm{~A}, 1 \mathrm{~B}, 2 \mathrm{~A}, 2 \mathrm{~B}, 3 \mathrm{~B}$, $3 \mathrm{D}, 4 \mathrm{~A}, 5 \mathrm{~A}, 5 \mathrm{~B}, 6 \mathrm{~A}, 6 \mathrm{~B}, 7 \mathrm{~A}, 7 \mathrm{~B}$ and $7 \mathrm{D})$ in the four experimental environments (Table S4). Some MATs clusters were found on chromosomes $1 \mathrm{1B}, 2 \mathrm{~B}, 6 \mathrm{~B}, 7 \mathrm{~A}$, and $7 \mathrm{~B}$. However, at the $P<10^{-5}$ level, no loci were found.

In turn, there were 159 MTAs loci $\left(P<10^{-4}\right)$ associated with $\mathrm{Cd}$ content in wheat grain on 19 chromosomes, except for chromosomes 2D and 7D in the four experimental environments (Table S4). There were MTAs clusters on chromosomes $1 \mathrm{~B}, 1 \mathrm{D}, 2 \mathrm{~B}, 3 \mathrm{~B}$, and $5 \mathrm{~B}$; further, 22 loci were found at the $P<10^{-5}$ level in E2, E3, and E4 (Table 4). Six loci explained more than $10 \%$ of phenotypic variation on chromosomes $1 \mathrm{~B}, 1 \mathrm{D}, 4 \mathrm{~A}, 4 \mathrm{~B}$, and $5 \mathrm{~A}$.

Finally, 99 MTAs $\left(P<10^{-4}\right)$ associated with $\mathrm{Pb}$ content were found on 16 chromosomes $(1 \mathrm{~A}, 1 \mathrm{~B}, 1 \mathrm{D}, 2 \mathrm{~A}$, $2 \mathrm{~B}, 3 \mathrm{~A}, 3 \mathrm{~B}, 4 \mathrm{~A}, 4 \mathrm{~B}, 5 \mathrm{~A}, 5 \mathrm{~B}, 6 \mathrm{~A}, 6 \mathrm{~B}, 7 \mathrm{~A}, 7 \mathrm{~B}$, and $7 \mathrm{D}$ in the four experimental environments (Table S4). MTAs clusters were detected mainly on chromosomes $1 \mathrm{~B}, 3 \mathrm{~B}$, $5 \mathrm{~B}, 6 \mathrm{~B}$, and $7 \mathrm{~B}$. Of these, 18 loci were identified at the $P<10^{-5}$ level, which involved chromosomes $1 \mathrm{~B}, 2 \mathrm{~B}, 3 \mathrm{~A}$, 5A, 6B, 7A, and 7B (Table 4). Five loci explained more than $10 \%$ of the phenotypic variation on chromosomes $5 \mathrm{~A}$ and $7 \mathrm{~B}$ in $\mathrm{E} 4$. The maximum contribution rate of Excalibur_c25090_830 on chromosome 7B was $13.43 \%$, and its P-value was as low as $8.11 \times 10^{-7}$. 
Table 3 Genome-wide Association Mapping results of beneficial mineral elements $\left(P<10^{-5}\right)$

\begin{tabular}{|c|c|c|c|c|c|c|}
\hline Trait & Env & SNP marker & Chr. & Position & $P$ & $R^{2}(\%)$ \\
\hline \multirow[t]{4}{*}{$\mathrm{Ca}$} & E4 & Excalibur_c41752_392 & $3 B$ & 67 & 4.70E-05 & 8.92 \\
\hline & E4 & BS00057451_51 & $3 B$ & 67 & 4.70E-05 & 8.92 \\
\hline & E4 & Kukri_c41797_393 & $5 \mathrm{~A}$ & 53 & 7.13E-06 & 10.97 \\
\hline & E4 & RFL_Contig2187_1025 & $5 \mathrm{~A}$ & 53 & 7.13E-06 & 10.97 \\
\hline \multirow[t]{14}{*}{$\mathrm{Mn}$} & E2 & wsnp_Ex_c4561_8184576 & 1B & 75 & $6.26 \mathrm{E}-05$ & 8.33 \\
\hline & E2 & wsnp_BF478690B_Ta_2_1 & 1B & 75 & $6.72 \mathrm{E}-05$ & 8.28 \\
\hline & E2 & Excalibur_c5218_75 & $1 \mathrm{~B}$ & 75 & $7.28 \mathrm{E}-05$ & 8.2 \\
\hline & E2 & IAAV2125 & 1B & 75 & $6.72 \mathrm{E}-05$ & 8.28 \\
\hline & E2 & IAAV6731 & 1B & 75 & $6.72 \mathrm{E}-05$ & 8.28 \\
\hline & E2 & JD_c3116_778 & 1B & 75 & $6.26 \mathrm{E}-05$ & 8.33 \\
\hline & E2 & Ra_c37969_549 & 1B & 75 & $8.25 \mathrm{E}-05$ & 8.05 \\
\hline & E2 & RAC875_C19014_725 & 1B & 75 & $6.26 \mathrm{E}-05$ & 8.33 \\
\hline & E2 & RAC875_rep_c112555_200 & $1 \mathrm{~B}$ & 75 & $6.72 \mathrm{E}-05$ & 8.28 \\
\hline & E2 & RAC875_rep_c119728_146 & $1 \mathrm{~B}$ & 75 & $8.92 \mathrm{E}-05$ & 7.97 \\
\hline & E2 & TA003725-0553 & 1B & 75 & $8.92 \mathrm{E}-05$ & 7.97 \\
\hline & E2 & BS00022619_51 & 1B & 75 & $7.72 \mathrm{E}-05$ & 8.14 \\
\hline & E2 & BS00022920_51 & $1 \mathrm{~B}$ & 75 & $6.72 \mathrm{E}-05$ & 8.28 \\
\hline & E2 & Tdurum_contig81102_102 & $1 \mathrm{~B}$ & 75 & $8.92 \mathrm{E}-05$ & 7.97 \\
\hline \multirow[t]{20}{*}{$\mathrm{Fe}$} & E2 & wsnp_Ex_c11913_19105189 & $5 \mathrm{~A}$ & 16 & 5.47E-05 & 8.75 \\
\hline & E2 & RAC875_rep_c112368_118 & $5 \mathrm{~A}$ & 16 & 7.57E-05 & 8.77 \\
\hline & E2 & Excalibur_c6326_77 & $6 \mathrm{~B}$ & 20 & $1.46 \mathrm{E}-05$ & 10.19 \\
\hline & E2 & RAC875_S114363_172 & $6 \mathrm{~B}$ & 22 & $5.14 \mathrm{E}-05$ & 8.87 \\
\hline & E3 & wsnp_Ex_c65899_64135487 & $7 D$ & 26 & $8.79 \mathrm{E}-05$ & 8.12 \\
\hline & E3 & wsnp_Ra_c8297_14095831 & $7 \mathrm{D}$ & 26 & $9.09 \mathrm{E}-05$ & 8.07 \\
\hline & E3 & D_contig11494_202 & $7 D$ & 26 & 7.70E-05 & 8.25 \\
\hline & E3 & D_F5XZDLF01ASSE2_190 & $7 D$ & 26 & 7.70E-05 & 8.25 \\
\hline & E3 & Ex_c25027_535 & $7 \mathrm{D}$ & 26 & 7.70E-05 & 8.25 \\
\hline & E3 & Excalibur_c833_1405 & $7 D$ & 26 & $8.85 \mathrm{E}-05$ & 8.1 \\
\hline & E3 & Kukri_rep_c103404_314 & $7 D$ & 26 & 7.70E-05 & 8.25 \\
\hline & E3 & BS00022449_51 & $7 D$ & 26 & $7.82 \mathrm{E}-05$ & 8.25 \\
\hline & E3 & BS00110124_51 & $7 D$ & 27 & 7.70E-05 & 8.25 \\
\hline & E3 & BS00110642_51 & $7 \mathrm{D}$ & 27 & 7.70E-05 & 8.25 \\
\hline & E3 & D_GB5Y7FA02IDDA9_183 & $7 D$ & 30 & 7.70E-05 & 8.25 \\
\hline & E3 & TA005377-1076 & $7 D$ & 32 & $6.50 \mathrm{E}-05$ & 8.43 \\
\hline & E4 & Excalibur_c19455_3496 & $7 \mathrm{~B}$ & 163 & $8.12 \mathrm{E}-08$ & 16.23 \\
\hline & E4 & Excalibur_c11062_582 & $7 \mathrm{~B}$ & 171 & $1.44 \mathrm{E}-05$ & 10.26 \\
\hline & E4 & Excalibur_c25090_830 & $7 \mathrm{~B}$ & 171 & $2.81 \mathrm{E}-06$ & 12.07 \\
\hline & E4 & RAC875_rep_c110526_229 & $7 \mathrm{~B}$ & 171 & $1.44 \mathrm{E}-05$ & 10.26 \\
\hline $\mathrm{Cu}$ & E2 & Excalibur_c29255_366 & $4 \mathrm{~B}$ & 104 & $8.12 \mathrm{E}-05$ & 8.91 \\
\hline
\end{tabular}


Table 3 (continued)

\begin{tabular}{|c|c|c|c|c|c|c|}
\hline Trait & Env & SNP marker & Chr. & Position & $P$ & $R^{2}(\%)$ \\
\hline \multirow[t]{16}{*}{$\mathrm{Zn}$} & E1 & BS00012036_51 & $2 B$ & 108 & $8.49 \mathrm{E}-05$ & 8 \\
\hline & E1 & BS00062691_51 & $4 \mathrm{~B}$ & 62 & $7.05 \mathrm{E}-06$ & 12.12 \\
\hline & E1 & CAP8_rep_c6942_227 & $7 \mathrm{~A}$ & 148 & $8.98 \mathrm{E}-05$ & 7.94 \\
\hline & E1 & BS00072941_51 & $7 B$ & 71 & $2.42 \mathrm{E}-05$ & 9.29 \\
\hline & E1 & BobWhite_c7907_657 & $7 B$ & 71 & $2.42 \mathrm{E}-05$ & 9.29 \\
\hline & E1 & Kukri_c78330_327 & $7 \mathrm{~B}$ & 71 & $3.23 \mathrm{E}-05$ & 8.99 \\
\hline & E1 & RFL_Contig2540_306 & $7 B$ & 71 & $2.42 \mathrm{E}-05$ & 9.29 \\
\hline & E1 & TA003961-0636 & $7 \mathrm{~B}$ & 71 & $2.42 \mathrm{E}-05$ & 9.29 \\
\hline & E1 & BS00095819_51 & $7 B$ & 72 & $6.62 \mathrm{E}-05$ & 8.25 \\
\hline & E1 & Tdurum_contig75931_1967 & $7 \mathrm{~B}$ & 72 & $6.62 \mathrm{E}-05$ & 8.25 \\
\hline & E1 & BobWhite_c40042_842 & $7 \mathrm{~B}$ & 101 & $8.98 \mathrm{E}-05$ & 7.94 \\
\hline & E3 & Ra_c19225_591 & $2 \mathrm{~B}$ & 130 & 4.19E-05 & 8.19 \\
\hline & E3 & wsnp_Ex_c9428_15641609 & $7 \mathrm{~A}$ & 159 & $3.63 \mathrm{E}-05$ & 8.42 \\
\hline & E3 & wsnp_Ex_c9428_15641639 & $7 \mathrm{~A}$ & 159 & $1.91 \mathrm{E}-05$ & 8.98 \\
\hline & E4 & Excalibur_c41752_392 & $3 B$ & 67 & 7.87E-07 & 13.25 \\
\hline & E4 & BS00057451_51 & $3 B$ & 67 & 7.87E-07 & 13.25 \\
\hline \multirow[t]{6}{*}{ Se } & E1 & Excalibur_rep_c93332_58 & $3 \mathrm{D}$ & 107 & $1.07 \mathrm{E}-05$ & 10.06 \\
\hline & E1 & BobWhite_c9622_723 & $3 \mathrm{D}$ & 113 & 3.93E-05 & 8.99 \\
\hline & E1 & wsnp_Ku_c21275_31007309 & $5 \mathrm{~A}$ & 83 & $3.46 \mathrm{E}-05$ & 8.77 \\
\hline & E2 & Tdurum_contig4974_355 & $4 \mathrm{~B}$ & 61 & $8.65 \mathrm{E}-05$ & 8.7 \\
\hline & E2 & wsnp_Ex_c14654_22713386 & $7 \mathrm{~A}$ & 42 & $2.66 \mathrm{E}-05$ & 9.68 \\
\hline & E2 & D_contig06359_118 & $7 D$ & 56 & $2.80 \mathrm{E}-05$ & 9.52 \\
\hline
\end{tabular}

$\mathrm{E} 1, \mathrm{E} 2, \mathrm{E} 3$ and $\mathrm{E} 4$ were same as the Table 1

\section{Stable MTAs loci at the Tai'an locations over 3 years}

A total of 66 stable MTAs loci $\left(P<10^{-4}\right)$ were found for $\mathrm{Ca}, \mathrm{Mn}, \mathrm{Cu}, \mathrm{Zn}, \mathrm{Se}, \mathrm{Cd}$, and $\mathrm{Pb}$ content in two or more of the tested environments (Table 5). Of these, five loci were detected for $\mathrm{Ca}$ content on chromosome 5A, and two major MATs explained more than $10 \%$ of the phenotypic variation. Eighteen stable MTAs for Mn content were identified on chromosomes 7A and 7B. Most were concentrated at the genetic position $75 \mathrm{cM}$ on chromosome 7B. A stable locus was found for $\mathrm{Cu}$ and $\mathrm{Zn}$ content on chromosomes 1A and 3B, and the BS00057451_51 locus on chromosome $3 \mathrm{~B}$ explained $13.25 \%$ of the phenotypic variation. Two stable loci on chromosomes $4 \mathrm{~B}$ and 7A were detected for Se content. The residue of stable MTAs loci was identified for $\mathrm{Cd}$ and $\mathrm{Pb}$ content. Five major loci were identified for $\mathrm{Pb}$ content.

\section{Multi-effect MTAs loci of mineral elements}

There were eight multi-effect MTAs loci for controlling more than one trait, mainly distributed on chromosomes 3B, 7B, and 5A (Table 6). There were two loci, BS00057451_51 and Excalibur_c41752_392, concurrently associated with $\mathrm{Ca}$ and $\mathrm{Zn}$ content on chromosome 3B. These two loci contributed to $13.25 \%$ of the variation in $\mathrm{Zn}$ content. One locus, Excalibur_c11062_582, simultaneously controlled Fe and Cd content on chromosome $7 \mathrm{~B}$, but the contribution to $\mathrm{Fe}$ content of this locus exhibited more than $10 \%$. Two loci, RAC875_rep_c110526_229 and Excalibur_c25090_830, were simultaneously associated with $\mathrm{Fe}, \mathrm{Cd}$, and $\mathrm{Pb}$ content on chromosome $7 \mathrm{~B}$. These two loci explained more than $10 \%$ of the variation in $\mathrm{Fe}$ and $\mathrm{Pb}$ content. The three loci were concentrated on the genetic position $171 \mathrm{cM}$ of chromosome 7B. There was one locus, Excalibur_c19455_3496, concurrently controlling Fe and $\mathrm{Pb}$ content, which accounted for more than $10 \%$ of the phenotypic variation on chromosome 7B. Two loci were identified for $\mathrm{Cd}$ and $\mathrm{Pb}$ content simultaneously on chromosome 5A, accounting for more than $10 \%$ phenotypic variation. 
Table 4 Genome-wide Association Mapping results of heavy metal elements $\left(P<10^{-5}\right)$

\begin{tabular}{|c|c|c|c|c|c|c|}
\hline Trait & Env & SNP marker & Chr. & Position & $P$ & $R^{2}(\%)$ \\
\hline \multirow[t]{22}{*}{$\mathrm{Cd}$} & E3 & Tdurum_contig44851_927 & $1 \mathrm{~B}$ & 162 & $3.23 \mathrm{E}-06$ & 11.77 \\
\hline & E2 & BS00083531_51 & $1 \mathrm{D}$ & 72 & $6.82 \mathrm{E}-05$ & 8.51 \\
\hline & E3 & Tdurum_contig44851_593 & $1 \mathrm{D}$ & 164 & $3.23 \mathrm{E}-06$ & 11.77 \\
\hline & E4 & RAC875_C9594_1289 & $1 \mathrm{D}$ & 65 & $9.63 \mathrm{E}-05$ & 8.20 \\
\hline & E4 & wsnp_Ex_rep_c108004_91402649 & $2 \mathrm{~A}$ & 168 & 4.77E-05 & 8.87 \\
\hline & E4 & GENE-0762_808 & $2 \mathrm{~A}$ & 168 & 4.64E-05 & 8.90 \\
\hline & E2 & CAP12_rep_c6956_169 & $2 B$ & 115 & 7.91E-05 & 8.32 \\
\hline & E2 & wsnp_Ex_c17538_26261053 & $2 B$ & 100 & 8.63E-05 & 8.25 \\
\hline & E2 & RAC875_c59545_122 & $2 B$ & 104 & $7.26 \mathrm{E}-05$ & 8.41 \\
\hline & E2 & Excalibur_rep_c66577_159 & $2 B$ & 107 & $5.00 \mathrm{E}-05$ & 8.82 \\
\hline & E3 & Tdurum_contig13489_292 & $4 \mathrm{~A}$ & 75 & 3.65E-06 & 11.65 \\
\hline & E3 & Kukri_c59197_207 & $4 \mathrm{~B}$ & 6 & 7.70E-06 & 11.05 \\
\hline & E3 & RAC875_c9572_588 & $4 \mathrm{~B}$ & 63 & $8.79 \mathrm{E}-05$ & 8.22 \\
\hline & E4 & wsnp_Ex_c28908_37989067 & $5 A$ & 27 & 4.85E-06 & 11.34 \\
\hline & E4 & wsnp_Ku_c1254_2498515 & $5 \mathrm{~A}$ & 27 & $1.43 \mathrm{E}-05$ & 10.16 \\
\hline & E4 & BS00033185_51 & $5 B$ & 174 & $9.69 \mathrm{E}-05$ & 8.14 \\
\hline & E3 & RAC875_rep_c69613_547 & $5 \mathrm{D}$ & 56 & $6.85 \mathrm{E}-05$ & 9.10 \\
\hline & E3 & wsnp_Ex_c7713_13153321 & $6 B$ & 92 & 4.09E-05 & 9.03 \\
\hline & E3 & Excalibur_c7713_272 & $7 \mathrm{~A}$ & 92 & $8.57 \mathrm{E}-05$ & 8.24 \\
\hline & E4 & Excalibur_c11062_582 & $7 B$ & 171 & $5.52 \mathrm{E}-05$ & 8.72 \\
\hline & E4 & Excalibur_c25090_830 & $7 \mathrm{~B}$ & 171 & $2.68 \mathrm{E}-05$ & 9.49 \\
\hline & E4 & RAC875_rep_c110526_229 & $7 B$ & 171 & $5.52 \mathrm{E}-05$ & 8.72 \\
\hline \multirow[t]{18}{*}{$\mathrm{Pb}$} & E3 & Ex_c4206_502 & $1 \mathrm{~B}$ & 108 & $6.88 \mathrm{E}-05$ & 8.48 \\
\hline & E2 & BS00083626_51 & $2 B$ & 173 & $9.90 \mathrm{E}-05$ & 7.94 \\
\hline & E4 & BS00022424_51 & $3 \mathrm{~A}$ & 141 & $5.65 \mathrm{E}-05$ & 8.76 \\
\hline & E3 & Tdurum_contig48760_112 & $5 A$ & 69 & 7.77E-05 & 8.37 \\
\hline & E4 & wsnp_Ex_c28908_37989067 & $5 A$ & 27 & $1.10 \mathrm{E}-05$ & 10.53 \\
\hline & E4 & wsnp_Ku_c1254_2498515 & $5 A$ & 27 & 3.89E-05 & 9.16 \\
\hline & E1 & Excalibur_c16961_85 & $6 B$ & 64 & $2.15 \mathrm{E}-05$ & 9.44 \\
\hline & E1 & BobWhite_c27318_380 & $6 B$ & 67 & $6.06 \mathrm{E}-05$ & 8.35 \\
\hline & E1 & Excalibur_c6416_1712 & $6 B$ & 67 & $6.07 \mathrm{E}-05$ & 8.35 \\
\hline & E1 & BobWhite_c36415_378 & $6 B$ & 67 & $9.21 \mathrm{E}-05$ & 7.90 \\
\hline & E1 & IACX203 & $6 B$ & 67 & $3.00 \mathrm{E}-05$ & 9.12 \\
\hline & E3 & Excalibur_c1215_334 & $7 \mathrm{~A}$ & 127 & $5.29 \mathrm{E}-05$ & 8.88 \\
\hline & E4 & RAC875_c57326_85 & $7 \mathrm{~B}$ & 134 & $8.71 \mathrm{E}-05$ & 8.30 \\
\hline & E4 & wsnp_Ex_c8400_14157060 & $7 B$ & 134 & $8.71 \mathrm{E}-05$ & 8.30 \\
\hline & E4 & Excalibur_c19455_3496 & $7 B$ & 163 & $9.11 \mathrm{E}-06$ & 10.81 \\
\hline & E4 & Excalibur_c11062_582 & $7 B$ & 171 & $1.90 \mathrm{E}-06$ & 12.47 \\
\hline & E4 & Excalibur_c25090_830 & $7 B$ & 171 & $8.11 \mathrm{E}-07$ & 13.43 \\
\hline & E4 & RAC875_rep_c110526_229 & $7 \mathrm{~B}$ & 171 & $1.90 \mathrm{E}-06$ & 12.47 \\
\hline
\end{tabular}

$\mathrm{E} 1, \mathrm{E} 2, \mathrm{E} 3$ and $\mathrm{E} 4$ were same as the Table 1

\section{Identification of stable MTAs and its alleles analysis in wheat accessions}

By screening the results (Tables S3 and S4), there were thirteen SNP markers identified for stable MTAs associated with $\mathrm{Ca}, \mathrm{Mn}, \mathrm{Zn}, \mathrm{Se}$ and $\mathrm{Pb}$ content (Table 7 and Fig. 1). Of which, the phenotypic value of $\mathrm{Ca}$ content associated with Kukri_c41797_393- TT on chromosome 5A was significantly higher than that associated with Kukri_c41797_393- CC across all four environments, which indicated that the contribution of Kukri_c41797_393-TT locus to Ca content was better than that of Kukri_c41797_393- CC locus, so did 
Table 5 Stable marker loci at Taian location of different years $\left(P<10^{-4}\right)$

\begin{tabular}{|c|c|c|c|c|c|}
\hline Trait & SNP marker & Chr. & Position & $P$ & $R^{2}(\%)$ \\
\hline \multirow[t]{5}{*}{$\mathrm{Ca}$} & Excalibur_c15014_1170 & $5 \mathrm{~A}$ & 50 & $3.71 \mathrm{E}-04$ & 6.76 \\
\hline & GENE-3167_70 & $5 \mathrm{~A}$ & 50 & $3.71 \mathrm{E}-04$ & 6.76 \\
\hline & Kukri_c2781_719 & $5 \mathrm{~A}$ & 50 & $3.71 \mathrm{E}-04$ & 6.76 \\
\hline & Kukri_c41797_393 & $5 \mathrm{~A}$ & 53 & 7.13E-06 & 10.97 \\
\hline & RFL_Contig2187_1025 & $5 \mathrm{~A}$ & 53 & 7.13E-06 & 10.97 \\
\hline \multirow[t]{18}{*}{$\mathrm{Mn}$} & BobWhite_rep_c66032_270 & $1 \mathrm{~B}$ & 71 & $2.38 \mathrm{E}-04$ & 6.99 \\
\hline & wsnp_BE443332B_Ta_2_2 & $1 \mathrm{~B}$ & 71 & $2.21 \mathrm{E}-04$ & 7.05 \\
\hline & wsnp_BE443930B_Ta_2_2 & $1 \mathrm{~B}$ & 71 & $2.82 \mathrm{E}-04$ & 6.81 \\
\hline & BS00022619_51 & $1 \mathrm{~B}$ & 75 & $7.72 \mathrm{E}-05$ & 8.14 \\
\hline & BS00022920_51 & $1 \mathrm{~B}$ & 75 & $6.72 \mathrm{E}-05$ & 8.28 \\
\hline & Excalibur_c5218_75 & $1 \mathrm{~B}$ & 75 & $7.28 \mathrm{E}-05$ & 8.20 \\
\hline & IAAV2125 & $1 \mathrm{~B}$ & 75 & $6.72 \mathrm{E}-05$ & 8.28 \\
\hline & IAAV6731 & $1 \mathrm{~B}$ & 75 & $6.72 \mathrm{E}-05$ & 8.28 \\
\hline & IAAV9005 & $1 \mathrm{~B}$ & 75 & $1.12 \mathrm{E}-04$ & 7.74 \\
\hline & JD_c3116_778 & $1 \mathrm{~B}$ & 75 & $6.26 \mathrm{E}-05$ & 8.33 \\
\hline & Ra_c37969_549 & $1 \mathrm{~B}$ & 75 & $8.25 \mathrm{E}-05$ & 8.05 \\
\hline & RAC875_C19014_725 & $1 \mathrm{~B}$ & 75 & $6.26 \mathrm{E}-05$ & 8.33 \\
\hline & RAC875_rep_c112555_200 & $1 \mathrm{~B}$ & 75 & $6.72 \mathrm{E}-05$ & 8.28 \\
\hline & RAC875_rep_c119728_146 & $1 \mathrm{~B}$ & 75 & $8.92 \mathrm{E}-05$ & 7.97 \\
\hline & TA003725-0553 & $1 \mathrm{~B}$ & 75 & $8.92 \mathrm{E}-05$ & 7.97 \\
\hline & Tdurum_contig81102_102 & $1 \mathrm{~B}$ & 75 & $8.92 \mathrm{E}-05$ & 7.97 \\
\hline & wsnp_BF478690B_Ta_2_1 & $1 \mathrm{~B}$ & 75 & $6.72 \mathrm{E}-05$ & 8.28 \\
\hline & wsnp_Ex_c4561_8184576 & $1 \mathrm{~B}$ & 75 & $6.26 \mathrm{E}-05$ & 8.33 \\
\hline $\mathrm{Cu}$ & RAC875_c23158_301 & $1 \mathrm{~A}$ & 77 & $6.31 \mathrm{E}-04$ & 6.28 \\
\hline $\mathrm{Zn}$ & BS00057451_51 & $3 B$ & 67 & 7.87E-07 & 13.25 \\
\hline \multirow[t]{2}{*}{ Se } & Tdurum_contig4974_355 & $4 \mathrm{~B}$ & 61 & $8.65 \mathrm{E}-05$ & 8.70 \\
\hline & wsnp_Ex_c14654_22713386 & $7 \mathrm{~A}$ & 42 & $2.66 \mathrm{E}-05$ & 9.68 \\
\hline \multirow[t]{13}{*}{$\mathrm{Cd}$} & Kukri_c105601_74 & $1 \mathrm{~B}$ & 51 & $2.15 \mathrm{E}-04$ & 7.29 \\
\hline & RAC875_c25101_644 & $1 \mathrm{~B}$ & 51 & $2.15 \mathrm{E}-04$ & 7.29 \\
\hline & BS00022255_51 & $1 \mathrm{~B}$ & 57 & $1.50 \mathrm{E}-04$ & 7.67 \\
\hline & Kukri_c18006_1568 & $1 \mathrm{~B}$ & 57 & $2.15 \mathrm{E}-04$ & 7.29 \\
\hline & RAC875_c8271_1352 & $1 \mathrm{~B}$ & 57 & $2.15 \mathrm{E}-04$ & 7.29 \\
\hline & RAC875_c8271_1469 & $1 \mathrm{~B}$ & 57 & $2.15 \mathrm{E}-04$ & 7.29 \\
\hline & RAC875_c8271_887 & $1 \mathrm{~B}$ & 57 & $2.15 \mathrm{E}-04$ & 7.29 \\
\hline & RAC875_rep_c96733_369 & $1 \mathrm{~B}$ & 57 & $2.15 \mathrm{E}-04$ & 7.29 \\
\hline & wsnp_Ku_c11987_19472688 & $1 \mathrm{~B}$ & 57 & $2.15 \mathrm{E}-04$ & 7.29 \\
\hline & wsnp_Ku_c11987_19473636 & $1 \mathrm{~B}$ & 58 & $2.06 \mathrm{E}-04$ & 7.35 \\
\hline & D_contig25392_201 & $1 \mathrm{~B}$ & 61 & $1.50 \mathrm{E}-04$ & 7.67 \\
\hline & Kukri_c23300_267 & $1 \mathrm{~B}$ & 61 & $2.15 \mathrm{E}-04$ & 7.29 \\
\hline & RAC875_c64253_435 & $5 \mathrm{~A}$ & 83 & 8.35E-04 & 5.92 \\
\hline
\end{tabular}

Table 5 (continued)

\begin{tabular}{|c|c|c|c|c|c|}
\hline Trait & SNP marker & Chr. & Position & $P$ & $R^{2}(\%)$ \\
\hline \multirow[t]{26}{*}{$\mathrm{Pb}$} & BS00022255_51 & $1 \mathrm{~B}$ & 57 & $1.28 \mathrm{E}-04$ & 7.90 \\
\hline & D_contig25392_201 & $1 \mathrm{~B}$ & 61 & $1.28 \mathrm{E}-04$ & 7.90 \\
\hline & RAC875_C9594_1289 & $1 \mathrm{D}$ & 65 & $5.83 \mathrm{E}-04$ & 6.37 \\
\hline & BS00091763_51 & $2 \mathrm{~A}$ & 167 & $5.30 \mathrm{E}-04$ & 6.41 \\
\hline & GENE-0762_808 & $2 \mathrm{~A}$ & 168 & $1.51 \mathrm{E}-04$ & 7.72 \\
\hline & $\begin{array}{l}\text { wsnp_Ex_rep_ } \\
\text { c108004_91402649 }\end{array}$ & $2 \mathrm{~A}$ & 168 & $2.24 \mathrm{E}-04$ & 7.31 \\
\hline & wsnp_Ex_c14162_22093694 & $2 \mathrm{~B}$ & 85 & $3.44 \mathrm{E}-04$ & 7.49 \\
\hline & BS00022424_51 & $3 \mathrm{~A}$ & 141 & $5.65 \mathrm{E}-05$ & 8.76 \\
\hline & RAC875_c17479_359 & $3 \mathrm{~A}$ & 93 & $8.02 \mathrm{E}-04$ & 5.99 \\
\hline & Tdurum_contig61465_781 & $4 \mathrm{~B}$ & 61 & $2.53 \mathrm{E}-04$ & 7.18 \\
\hline & wsnp_Ex_c28908_37989067 & $5 \mathrm{~A}$ & 27 & $1.10 \mathrm{E}-05$ & 10.53 \\
\hline & wsnp_Ku_c1254_2498515 & $5 \mathrm{~A}$ & 27 & $3.89 \mathrm{E}-05$ & 9.16 \\
\hline & BobWhite_c46416_247 & $5 B$ & 183 & $3.24 \mathrm{E}-04$ & 6.92 \\
\hline & BS00067074_51 & $5 B$ & 183 & $1.29 E-04$ & 7.88 \\
\hline & CAP7_c8713_356 & $5 B$ & 183 & 1.29E-04 & 7.88 \\
\hline & Kukri_c4594_825 & $5 B$ & 183 & $3.24 \mathrm{E}-04$ & 6.92 \\
\hline & RAC875_C1035_65 & $5 B$ & 183 & $1.29 \mathrm{E}-04$ & 7.88 \\
\hline & Tdurum_contig60189_310 & $5 B$ & 183 & $1.30 \mathrm{E}-04$ & 7.89 \\
\hline & BS00068775_51 & $5 B$ & 184 & $1.65 \mathrm{E}-04$ & 7.72 \\
\hline & CAP7_c3697_87 & $6 \mathrm{~B}$ & 86 & $1.31 \mathrm{E}-04$ & 7.87 \\
\hline & Tdurum_contig68217_361 & $6 \mathrm{~B}$ & 86 & 1.31E-04 & 7.87 \\
\hline & BobWhite_c17095_237 & $7 \mathrm{~A}$ & 136 & $3.65 \mathrm{E}-04$ & 6.80 \\
\hline & Excalibur_c19455_3496 & $7 \mathrm{~B}$ & 163 & $9.11 \mathrm{E}-06$ & 10.81 \\
\hline & Excalibur_c11062_582 & $7 \mathrm{~B}$ & 171 & 1.90E-06 & 12.47 \\
\hline & Excalibur_c25090_830 & $7 \mathrm{~B}$ & 171 & $8.11 \mathrm{E}-07$ & 13.43 \\
\hline & RAC875_rep_c110526_229 & $7 \mathrm{~B}$ & 171 & $1.90 \mathrm{E}-06$ & 12.47 \\
\hline
\end{tabular}

the contribution of RFL_Contig2187_1025 locus to Ca content.

For Mn content, five SNP makers were detected on chromosome 1B (Table 7). The contribution of AA allele to Mn content was significantly higher than that of GG allele for BS00022619 51 and BS00022920 51 markers, but for Excalibur_c5218_75 marker, the GG allele was better than the AA allele for improving the Mn content. The contribution of CC allele of IAAV2125 and IAAV6731 to Mn content showed better than that of AA allele.

Only one stable marker BS00057451_51 was found on chromosome 3B for $\mathrm{Zn}$ content, and the contribution of 
Table 6 Multi-effect loci associated with mineral elements $\left(P<10^{-5}\right)$

\begin{tabular}{|c|c|c|c|c|c|}
\hline Trait & SNP marker & Chr. & Position & $P$ & $R^{2}(\%)$ \\
\hline $\mathrm{Ca}$ & BS00057451_51 & $3 B$ & 67 & 4.70E-05 & 8.92 \\
\hline $\mathrm{Zn}$ & BS00057451_51 & $3 B$ & 67 & 7.87E-07 & 13.25 \\
\hline $\mathrm{Ca}$ & Excalibur_c41752_392 & $3 B$ & 67 & 4.70E-05 & 8.92 \\
\hline $\mathrm{Zn}$ & Excalibur_c41752_392 & $3 B$ & 67 & 7.87E-07 & 13.25 \\
\hline $\mathrm{Fe}$ & Excalibur_c11062_582 & $7 \mathrm{~B}$ & 171 & $1.44 \mathrm{E}-05$ & 10.26 \\
\hline $\mathrm{Cd}$ & Excalibur_c11062_582 & $7 \mathrm{~B}$ & 171 & $5.52 \mathrm{E}-05$ & 8.72 \\
\hline $\mathrm{Fe}$ & RAC875_rep_c110526_229 & $7 \mathrm{~B}$ & 171 & $1.44 \mathrm{E}-05$ & 10.26 \\
\hline $\mathrm{Cd}$ & RAC875_rep_c110526_229 & $7 \mathrm{~B}$ & 171 & $5.52 \mathrm{E}-05$ & 8.72 \\
\hline $\mathrm{Pb}$ & RAC875_rep_c110526_229 & $7 \mathrm{~B}$ & 171 & $1.90 \mathrm{E}-06$ & 12.47 \\
\hline $\mathrm{Fe}$ & Excalibur_c25090_830 & $7 \mathrm{~B}$ & 171 & $2.81 \mathrm{E}-06$ & 12.07 \\
\hline $\mathrm{Cd}$ & Excalibur_c25090_830 & $7 \mathrm{~B}$ & 171 & $2.68 \mathrm{E}-05$ & 9.49 \\
\hline $\mathrm{Pb}$ & Excalibur_c25090_830 & $7 \mathrm{~B}$ & 171 & $8.11 \mathrm{E}-07$ & 13.43 \\
\hline $\mathrm{Fe}$ & Excalibur_c19455_3496 & $7 \mathrm{~B}$ & 163 & $8.12 \mathrm{E}-08$ & 16.23 \\
\hline $\mathrm{Pb}$ & Excalibur_c19455_3496 & $7 \mathrm{~B}$ & 163 & $9.11 \mathrm{E}-06$ & 10.81 \\
\hline $\mathrm{Cd}$ & wsnp_Ex_c28908_37989067 & $5 \mathrm{~A}$ & 27 & 4.85E-06 & 11.34 \\
\hline $\mathrm{Pb}$ & wsnp_Ex_c28908_37989067 & $5 \mathrm{~A}$ & 27 & 1.10E-05 & 10.53 \\
\hline $\mathrm{Cd}$ & wsnp_Ku_c1254_2498515 & $5 \mathrm{~A}$ & 27 & $1.43 \mathrm{E}-05$ & 10.16 \\
\hline $\mathrm{Pb}$ & wsnp_Ku_c1254_2498515 & $5 \mathrm{~A}$ & 27 & $3.89 \mathrm{E}-05$ & 9.16 \\
\hline
\end{tabular}

GG allele was significantly better than that of AA allele (Table 7 and Fig. 1).

For Se content, two markers, Tdurum_contig4974_355 and wsnp_Ex_c14654_22713386, were found on chromosome $4 \mathrm{~B}$ and 7A, respectively (Table 7 and Fig. 1). The contribution of Tdurum_contig4974_355-TT allele showed significantly better than that of Tdurum contig4974_355-CC for Se content, but for wsnp_Ex c14654_22713386 marker, the CC allele was better than the TT allele.

Three stable SNP markers were identified on chromosome $7 \mathrm{~B}$ for heavy metal $\mathrm{Pb}$ content (Table 7 and Fig. 1). The contributions of the alleles GG, CC and CC showed better than that of the alleles AA, TT and AA for Excalibur_c19455_3496, Excalibur_c25090_830 and Excalibur_ c11062_582 to reduce the $\mathrm{Pb}$ content, respectively. Most interestingly, these three SNP markers were also associated with Fe content.

So a pyramid analysis of the alleles of these different stable SNP markers were further studied in wheat accessions, seven accessions were found with high beneficialmineral-element contents and low heavy-metal-element contents (Table 8). Of which, five accessions (B111, B117,

Table 7 The allele analysis of stable MTAs in different mineral elements

\begin{tabular}{|c|c|c|c|c|c|c|}
\hline \multirow{2}{*}{$\begin{array}{l}\text { Trait } \\
\mathrm{Ca}\end{array}$} & \multirow{2}{*}{$\begin{array}{l}\text { SNP marker } \\
\text { Kukri_c41797_393 }\end{array}$} & \multirow{2}{*}{$\begin{array}{l}\text { Chr. } \\
5 \mathrm{~A}\end{array}$} & \multirow{2}{*}{$\begin{array}{l}\text { Position } \\
53\end{array}$} & \multirow{2}{*}{$\begin{array}{l}\text { Allele } \\
T / C\end{array}$} & \multicolumn{2}{|c|}{ Mean $(\mu \mathrm{g} / \mathrm{mL})$} \\
\hline & & & & & $\mathrm{CC}$ & $1807.8624 b$ \\
\hline & & & & & $\pi$ & $2084.2116 a$ \\
\hline \multirow[t]{2}{*}{$\mathrm{Ca}$} & RFL_Contig2187_1025 & $5 \mathrm{~A}$ & 53 & $\mathrm{~T} / \mathrm{C}$ & CC & $1807.8624 b$ \\
\hline & & & & & $\mathrm{TT}$ & $2084.2116 a$ \\
\hline \multirow[t]{2}{*}{$\mathrm{Mn}$} & Excalibur_c5218_75 & $1 \mathrm{~B}$ & 75 & $A / G$ & $\mathrm{AA}$ & $110.2797 b$ \\
\hline & & & & & GG & $118.0122 \mathrm{a}$ \\
\hline \multirow[t]{2}{*}{$\mathrm{Mn}$} & IAAV2125 & $1 \mathrm{~B}$ & 75 & $\mathrm{~A} / \mathrm{C}$ & $\mathrm{AA}$ & $110.2636 b$ \\
\hline & & & & & CC & $117.9426 \mathrm{a}$ \\
\hline \multirow[t]{2}{*}{$\mathrm{Mn}$} & IAAV6731 & $1 \mathrm{~B}$ & 75 & $\mathrm{~A} / \mathrm{C}$ & AA & $110.2636 b$ \\
\hline & & & & & CC & $117.9426 a$ \\
\hline \multirow[t]{2}{*}{$\mathrm{Mn}$} & BS00022619_51 & $1 \mathrm{~B}$ & 75 & $A / G$ & $\mathrm{AA}$ & $118.0122 \mathrm{a}$ \\
\hline & & & & & GG & $110.1032 b$ \\
\hline \multirow[t]{2}{*}{$\mathrm{Mn}$} & BS00022920_51 & $1 \mathrm{~B}$ & 75 & $\mathrm{~A} / \mathrm{G}$ & AA & $117.9426 a$ \\
\hline & & & & & GG & $110.2636 b$ \\
\hline \multirow[t]{2}{*}{$\mathrm{Zn}$} & BS00057451_51 & $3 B$ & 67 & $A / G$ & AA & $93.5820 \mathrm{~b}$ \\
\hline & & & & & GG & $107.1229 a$ \\
\hline \multirow[t]{2}{*}{ Se } & Tdurum_contig4974_355 & $4 \mathrm{~B}$ & 61 & $\mathrm{~T} / \mathrm{C}$ & CC & $0.0003 b$ \\
\hline & & & & & $\mathrm{TT}$ & $0.0007 \mathrm{a}$ \\
\hline \multirow[t]{2}{*}{ Se } & wsnp_Ex_c14654_22713386 & $7 \mathrm{~A}$ & 42 & $\mathrm{~T} / \mathrm{C}$ & CC & $0.0007 \mathrm{a}$ \\
\hline & & & & & $\mathrm{TT}$ & $0.0003 b$ \\
\hline \multirow[t]{2}{*}{$\mathrm{Pb}$} & Excalibur_c19455_3496 & $7 B$ & 163 & $A / G$ & AA & $0.0105 \mathrm{a}$ \\
\hline & & & & & GG & $0.0003 b$ \\
\hline \multirow[t]{2}{*}{$\mathrm{Pb}$} & Excalibur_c25090_830 & $7 B$ & 171 & $\mathrm{~T} / \mathrm{C}$ & TT & $0.0113 a$ \\
\hline & & & & & CC & $0.0003 b$ \\
\hline \multirow[t]{2}{*}{$\mathrm{Pb}$} & Excalibur_c11062_582 & $7 \mathrm{~B}$ & 171 & $\mathrm{~A} / \mathrm{C}$ & $\mathrm{AA}$ & $0.1469 a$ \\
\hline & & & & & CC & $0.0557 \mathrm{~b}$ \\
\hline
\end{tabular}




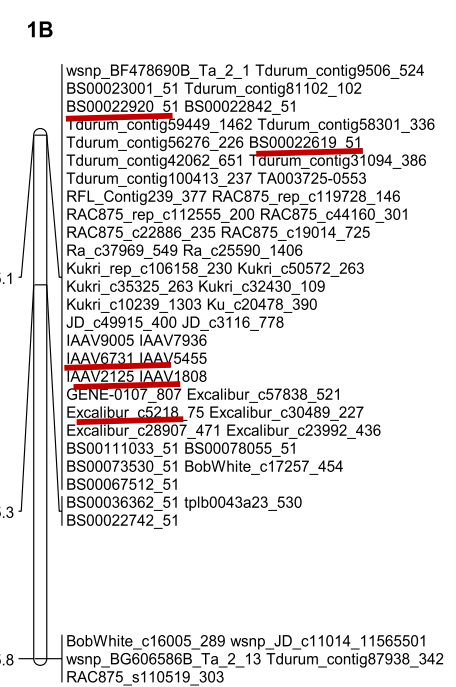

3B

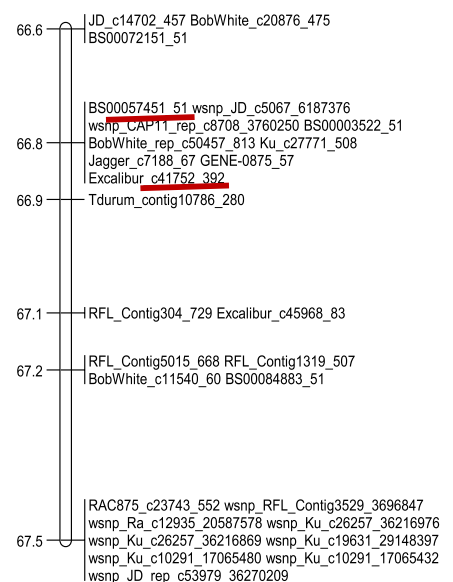

$4 B$

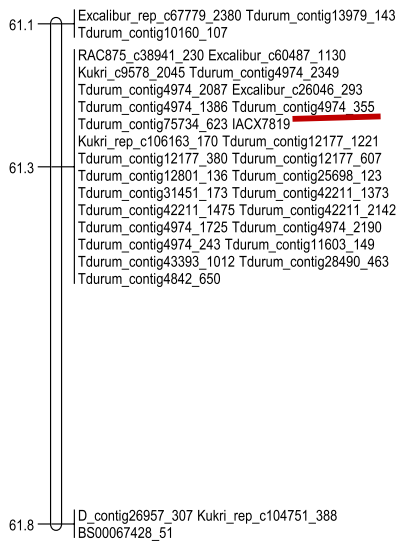

$5 \mathrm{~A} 1$

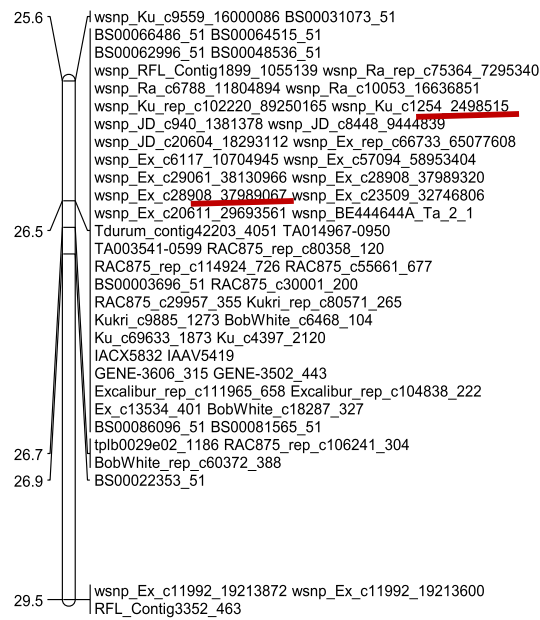

25.6 Wsnp_Ku_c9559_16000086 BS00031073_51 BS000662996 51 BS BS00064515_51

wsnp_RFL_Contig1899 1055139 wsnp_Ra_rep_c75364_7295340

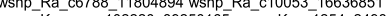
wsn_Ku_rep_c102220 89250165 w_ns_Ku_c1254 2498515 wsnp_J_c940-138 18293112 wsD._Ex rep c66733 6507760 wsnp_Ex_c6117 10704945 wsnp Ex c57094_58953404 wsnp_Ex_c29061_38130966 wsnp_Ex_c28908_37989320 wsnp_Ex_c28908

26.5 wsnp_Ex_c20611_29693561 wsnp_BE444644A_ Ta 2 1 Tdurum_contig42203_4051 TA014967-0950 RAC875_rep_c114924_726 RAC875_c55661_677 BS00003696_51 RAC875_c30001_200 (8) 875_c29957_355 Kukri_rep_c80571_265 Ku_c69633_1873 Ku_c4397_2120 GENE-3606 315 GENE-3502 443 Excalibur_rep_c111965_658 Excalibur_rep_c104838_222 Ex_c13534_401 BobWhite_c18287_ tplb0029e02_1186 RAC875_rep_106241 304 BSo0022353_51

$29.5-\left.\right|_{\text {RFL_Contig3352_463 }} ^{\text {wsnp_Ex } 119213872}$ wsnp_Ex_c11992_19213600

$3 A$

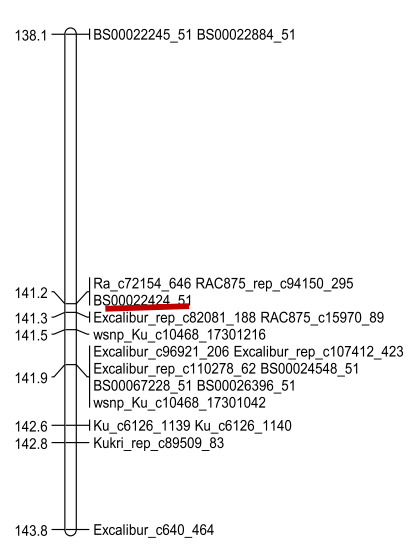

7B1

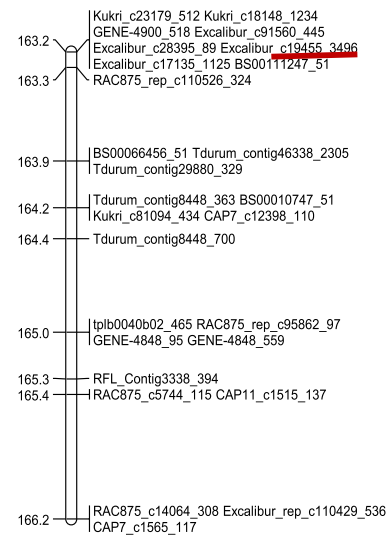

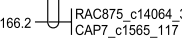

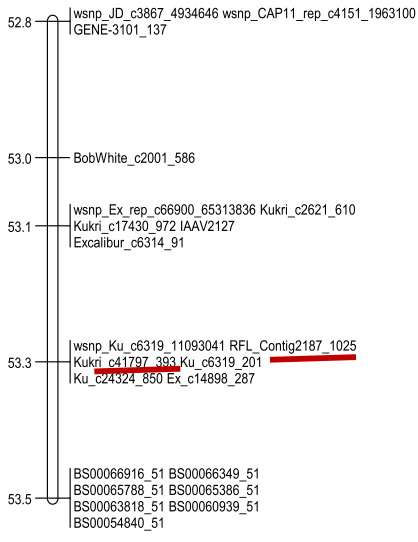

7B2

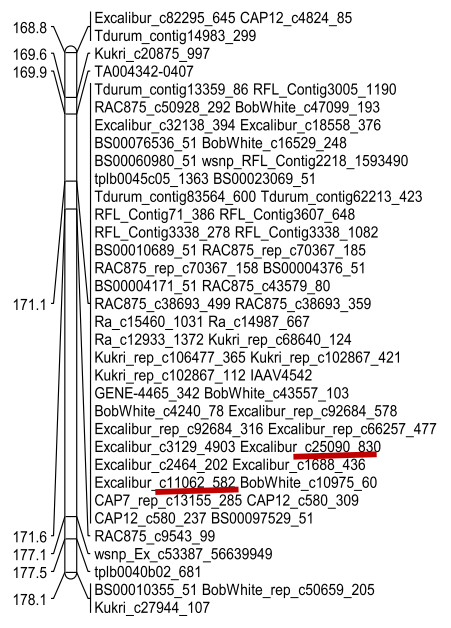

Fig. 1 The position of major stable MTAs in the chromosome maps 
Table 8 A pyramid analysis of different QTL alleles in certain wheat accessions

\begin{tabular}{|c|c|c|c|c|}
\hline Wheat accessions & Mineral elements & SNP marker & Allele & Content (ug/mL) \\
\hline \multirow[t]{5}{*}{ B111 } & $\mathrm{Ca}$ & Kukri_c41797_393 & TT & 2903.4414 \\
\hline & $\mathrm{Ca}$ & RFL_Contig2187_1025 & TT & \\
\hline & $\mathrm{Zn}$ & BS00057451_51 & GG & 139.8417 \\
\hline & $\mathrm{Pb}$ & Excalibur_c19455_3496 & GG & 0 \\
\hline & $\mathrm{Pb}$ & Excalibur_c11062_582 & CC & \\
\hline \multirow[t]{4}{*}{ B117 } & $\mathrm{Ca}$ & Kukri_c41797_393 & $\mathrm{TT}$ & 2356.8505 \\
\hline & $\mathrm{Ca}$ & RFL_Contig2187_1025 & $\mathrm{TT}$ & \\
\hline & $\mathrm{Fe}$ & Excalibur_c19455_3496 & AA & 185.3263 \\
\hline & $\mathrm{Pb}$ & Excalibur_c11062_582 & $\mathrm{CC}$ & 0 \\
\hline \multirow[t]{8}{*}{ B148 } & $\mathrm{Mn}$ & Excalibur_c5218_75 & GG & 148.6161 \\
\hline & $\mathrm{Mn}$ & IAAV2125 & $\mathrm{CC}$ & \\
\hline & $\mathrm{Mn}$ & IAAV6731 & CC & \\
\hline & $\mathrm{Mn}$ & BS00022619_51 & $A A$ & \\
\hline & $\mathrm{Mn}$ & BS00022920_51 & $A A$ & \\
\hline & $\mathrm{Zn}$ & BS00057451_51 & GG & 100.9967 \\
\hline & $\mathrm{Pb}$ & Excalibur_c19455_3496 & GG & \\
\hline & $\mathrm{Pb}$ & Excalibur_c11062_582 & $\mathrm{CC}$ & 0 \\
\hline \multirow[t]{10}{*}{ B46 } & $\mathrm{Mn}$ & Excalibur_c5218_75 & GG & 140.9914 \\
\hline & $\mathrm{Mn}$ & IAAV2125 & CC & \\
\hline & $\mathrm{Mn}$ & IAAV6731 & CC & \\
\hline & $\mathrm{Mn}$ & BS00022619_51 & $A A$ & \\
\hline & $\mathrm{Mn}$ & BS00022920_51 & AA & \\
\hline & $\mathrm{Zn}$ & BS00057451_51 & GG & 107.564 \\
\hline & $\mathrm{Se}$ & Tdurum_contig4974_355 & $\mathrm{TT}$ & 0.0016 \\
\hline & $\mathrm{Se}$ & wsnp_Ex_c14654_22713386 & $\mathrm{CC}$ & \\
\hline & $\mathrm{Pb}$ & Excalibur_c19455_3496 & GG & 0 \\
\hline & $\mathrm{Pb}$ & Excalibur_c11062_582 & CC & \\
\hline \multirow[t]{6}{*}{ B67 } & $\mathrm{Fe}$ & Excalibur_c19455_3496 & AA & 364.4478 \\
\hline & $\mathrm{Fe}$ & Excalibur_c25090_830 & CC & \\
\hline & $\mathrm{Fe}$ & Excalibur_c11062_582 & $A A$ & \\
\hline & $\mathrm{Se}$ & Tdurum_contig4974_355 & $\mathrm{TT}$ & 0.0016 \\
\hline & $\mathrm{Se}$ & wsnp_Ex_c14654_22713386 & $\mathrm{CC}$ & \\
\hline & $\mathrm{Pb}$ & Excalibur_c25090_830 & $\mathrm{CC}$ & 0 \\
\hline \multirow[t]{5}{*}{ B70 } & $\mathrm{Fe}$ & Excalibur_c25090_830 & TT & 358.5477 \\
\hline & $\mathrm{Se}$ & Tdurum_contig4974_355 & $\mathrm{TT}$ & 0.0019 \\
\hline & $\mathrm{Se}$ & wsnp_Ex_c14654_22713386 & CC & 0 \\
\hline & $\mathrm{Pb}$ & Excalibur_c19455_3496 & GG & \\
\hline & $\mathrm{Pb}$ & Excalibur_c11062_582 & $\mathrm{CC}$ & \\
\hline \multirow[t]{7}{*}{ B114 } & $\mathrm{Mn}$ & Excalibur_c5218_75 & GG & 156.3802 \\
\hline & $\mathrm{Mn}$ & IAAV2125 & $\mathrm{CC}$ & \\
\hline & $\mathrm{Mn}$ & IAAV6731 & CC & \\
\hline & $\mathrm{Mn}$ & BS00022619_51 & AA & \\
\hline & $\mathrm{Mn}$ & BS00022920_51 & AA & \\
\hline & $\mathrm{Pb}$ & Excalibur_c19455_3496 & GG & 0 \\
\hline & $\mathrm{Pb}$ & Excalibur_c11062_582 & $\mathrm{CC}$ & \\
\hline
\end{tabular}


B148, B46 and B67) contained more than one beneficial mineral elements with high content without $\mathrm{Pb}$ content. These results provided good wheat resources and elite alleles with beneficial mineral elements in wheat breeding.

\section{Prediction of candidate genes for important loci for mineral elements}

In all, 16 new candidate genes were predicted for 17 important loci for mineral elements (Table S5). On chromosome 3B, two candidate genes, TraesCS3B02G307600.1 and TraesCS3B02G307400, were found for BS00057451_51 and Excalibur_c41752_392, respectively, which involved $\mathrm{Zn}$ and $\mathrm{Ca}$ content. Their functions were primarily involved in metal ion binding, calcium ion binding, ATP binding, ATPase activity, DNA binding, RNA binding, and protein kinase activity.

There was one candidate gene for Se content predicted on chromosome 3D, TraesCS3D02G201900, whose function is modification-dependent protein binding in wheat. Furthermore, metal ion binding and calcium ion binding of this gene were found in Arabidopsis and rice, respectively.

Three candidate genes, TraesCS5A02G256700.1, TraesCS5A01G256800.1, and TraesCS5A02G257000.1, were identified on chromosome $5 \mathrm{~A}$ for $\mathrm{Ca}$ content. The gene TraesCS5A02G256700.1 for Kukri_c41797_393 had ribosomal small subunit biogenesis and exportation from the nucleus in wheat, but metal ion binding, $\mathrm{Ca}$ ion binding, and $\mathrm{Ca}$ transmembrane activity in Arabidopsis. The TraesCS5A02G257000.1 gene functions in $\mathrm{Zn}$-ion binding, $\mathrm{Zn}$-ion transmembrane transporter activity, and metal ion binding in Arabidopsis and rice.

For $\mathrm{Zn}$ content, there was one candidate gene TraesCS7B02G142200 for BobWhite_c7907_657 was identified on chromosome $7 \mathrm{~B}$, whose function was related to DNA binding and metal ion binding in wheat.

For Fe content, two candidate genes, TraesCS6B02G029300 and TraesCS6B02G029200, for Excalibur_c6326_77 were identified on chromosome 6B. The functions of these two genes are mainly related to $\mathrm{Ca}$-dependent $\mathrm{Ca}$-ion binding, $\mathrm{Fe}-$ ion binding, ATP binding, and DNA binding in Arabidopsis.

On chromosome 7B, two candidate genes were identified for Fe content which were also involved in $\mathrm{Cd}$ and $\mathrm{Pb}$ content. The gene TraesCS7B02G480300 for Excalibur_c19455_3496 was mainly related to Fe-ion binding, Fe- and $\mathrm{Zn}$-ion transmembrane transporter activity, $\mathrm{Ca}$ ion binding, metal ion binding, and metal-ion transmembrane transporter activity in Hordeum vulgare, Oryza sativa, and Arabidopsis. This gene mainly participates in the biological processes of $\mathrm{Fe}, \mathrm{Zn}$, and metal ion transport. The other gene, TraesCS7B02G478200, was also mainly related to Fe-ion binding, metal ion binding, $\mathrm{Zn}$ ion binding, and Ca-ion binding in Oryza, Arabidopsis, and Triticum urartu. In Arabidopsis, this gene shows a biological response to $\mathrm{Cd}$ ions as an aspartic-type endopeptidase activity.

Five candidate genes were found for $\mathrm{Cd}$ content on chromosomes $1 \mathrm{~B}, 1 \mathrm{D}, 4 \mathrm{~A}, 4 \mathrm{~B}$, and $5 \mathrm{~A}$. The gene TraesCS1B02G474800 for Tdurum_contig44851_927 was mainly related to $\mathrm{Cu}$-ion binding and metal ion binding in Oryza and Arabidopsis. The gene mainly participates in lignin breakdown. The function of TraesCS1D02G448700 mainly involves Fe-ion binding, Ca-ion binding, metal ion binding, and Mg-ion binding in Arabidopsis and Oryza. One of the biological processes it influences is the response to $\mathrm{Cd}$ ions. The gene TraesCS4B02G004800 functions in $\mathrm{Cu}$-ion binding, metal ion binding, four $\mathrm{Fe}$ and four $\mathrm{S}$ cluster binding, and the management of ion transmembrane transporter activity in Oryza and Arabidopsis. However, the gene TraesCS5A02G014600 affected $\mathrm{Cd}$ and $\mathrm{Pb}$ content involved in $\mathrm{Zn}$-ion binding, and cation- and Mn-transmembrane transporter activity, Mg-ion binding, and metal ion binding.

\section{Discussion}

Metal elements include beneficial and harmful elements; of which, beneficial elements are important for maintaining good human health. However, to ensure food safety, heavy metals should be avoided. In addition to the agronomic practices for biofortification of $\mathrm{Zn}, \mathrm{Fe}$, and Se, genetic improvement of these elements has become important. Previous studies have identified some QTL/gene loci for $\mathrm{Zn}$, Se, and Fe content involving all 21 wheat chromosomes [13, 15-18, 27, 31-38]; however, in our study, MTAs loci for $\mathrm{Ca}, \mathrm{Zn}, \mathrm{Fe}, \mathrm{Se}, \mathrm{Mn}$, and $\mathrm{Cu}$ content were found on 20 chromosomes, i.e. all except for chromosome 6D. Previously, the important QTLs detected for Zn, Fe, and Se were located on chromosomes 7B, 4A, 3B, 2D, 5A, 5B, 6A, and $7 \mathrm{~A}$ [13]. In this study, chromosomes $1 \mathrm{~B}, 3 \mathrm{~B}, 3 \mathrm{D}, 4 \mathrm{~B}, 5 \mathrm{~A}$, $6 \mathrm{~B}, 7 \mathrm{~B}$, and 7D were important for MTAs loci associated with $\mathrm{Ca}, \mathrm{Zn}, \mathrm{Fe}, \mathrm{Se}, \mathrm{Mn}$, and $\mathrm{Cu}$ content. Comparing these, common chromosomes $3 \mathrm{~B}, 5 \mathrm{~A}$, and $7 \mathrm{~B}$ were found to play important roles in regulating $\mathrm{Ca}, \mathrm{Fe}$, and $\mathrm{Zn}$ concentrations in the wheat grain, and to contain some important genes.

Using bioinformatics, the candidate genes (mRNA_2.1, mRNA_3.1, mRNA_10.1, mRNA_23.1, mRNA_24.1, and mRNA_32.1; mRNA_11.1, mRNA_34.1, mRNA_42.1, and mRNA_44.1) associated with $\mathrm{Zn}$ content were identified in the physical regions of 3BS $(723,504,241$ to $723,611,488$ ) (first six genes) and 5AL (462,763,758 to $468,582,184$ ) (last four genes) [21]. This study identified two candidate genes, TraesCS3B02G307600.1, with the physical region from 493,655,348 to $493,657,938$ 
and TraesCS3B02G307400 with the physical region from 493,648,449 to 493,653,177, on chromosome 3B, which is associated with grain $\mathrm{Ca}$ and $\mathrm{Zn}$ concentrations. By comparing their physical positions, we found that these two genes are different from the above genes located upstream of previously published genes. These published 3BS genes were found to belong to the mitogen-activated protein kinase (MAPK) family of genes involved in kinase activity, leading to protein phosphorylation, which in turn assists in the desired molecular function in various biological processes [13]. MAPKs are involved in $\mathrm{Zn}$ uptake and transport through signalling pathways. The mRNA_32.1 gene encodes a suppressor of the white apricot protein associated with $\mathrm{Zn}$ concentration in chickpea seeds [39], an RNA-binding protein involved in RNA processing [13]. In our study, according to the putative functions of these two new candidate genes, they are seemingly also involved in protein kinase activity and RNA binding; although primarily they are involved in metal ion binding, Ca-ion binding, ATP binding, and ATPase activity, indicating that they are involved in $\mathrm{Ca}$ and $\mathrm{Zn}$ uptake and transport. On chromosome $5 \mathrm{AL}$, the last four genes encode TaMTP proteins, which are directly or indirectly involved in $\mathrm{Zn}$ biofortification, and their functions are mainly involved in DNA binding, $\mathrm{Zn} / \mathrm{Fe}$ binding, and protein dimerisation [13, 21], However, this study identified three candidate genes, TraesCS5A02G256700.1, TraesCS5A01G256800.1, and TraesCS5A02G257000.1 on chromosome 5A for Ca content in wheat grain; further, their physical region is from $472,274,579$ to $472,347,557$, which is different from the physical position of the published genes. Their functions are primarily involved in $\mathrm{Ca}$-ion binding, $\mathrm{Zn}$-ion binding, metal ion binding, $\mathrm{Zn}$ - and iron-transmembrane transporter activity, ATP binding, and protein kinase activity. Additionally, some researchers have found that chromosome $5 \mathrm{~A}$ plays an important role in regulating grain $\mathrm{Cu}$ concentration [27, 34]. Therefore, these new candidate genes identified on chromosomes $3 \mathrm{~B}$ and $5 \mathrm{~A}$ are suitable for further molecular genetic research.

On chromosome 7B, two major QTLs for controlling Zn content were identified using DArT-seq [15]. These genes encode the kinase-like superfamily, which catalyses phosphorylation processes in which some protein structures are $\mathrm{Zn}$ related [15]. In this study, a new candidate gene, TraesCS7B02G142200, was predicted to be involved in DNA binding and metal ion binding in wheat. These results indicate that the genes are controlling $\mathrm{Zn}$ concentration on chromosome 7B.

Regarding ferritin, previous studies found genes involved in vacuolar iron transporters and transporter-like protein, such as TaFer1 and TaFer2 on homologous groups 5 and 4 in wheat, respectively [40]. Another wheat gene relevant to biofortification is the major grain protein gene $\mathrm{Gpc} 1$ on chromosome 6B, which also affects $\mathrm{Zn}$ and Fe concentrations in the grain $[13,41]$. as it can regulate the expression of several genes involved in the export and transport of $\mathrm{Zn}$ and Fe into the grain through the phloem [42]. This study predicted three candidate genes, TraesCS6B02G029300, TraesCS7B02G480300, and TraesCS7B02G478200, with physical regions from $17,703,175$ to $17,704,083$, $734,259,844$ to $734,274,042$, and $733,527,458$ to $733,530,221$, respectively, which are primarily involved in $\mathrm{Fe}$ - and $\mathrm{Ca}$-ion binding, metal ion binding, $\mathrm{Zn}$-ion binding, ATP binding, ATPase activity, and DNA binding. Thus, these genes are important for improving Fe concentration, a finding that warrants further research.

The accumulation of heavy metals (e.g. Cd, As, and $\mathrm{Pb}$ ) is a complex quantitative trait controlled by multiple genes. Most previous studies on the mechanisms of $\mathrm{Cd}$ accumulation have focused on rice, maize, and A. thaliana. A major QTL was mapped to translate Cd from roots to shoots at the seedling stage in rice [43]. By means of GWAS, a single strong peak of SNPs associated with leaf $\mathrm{Cd}$ accumulation was identified in A. thaliana [44]. In maize, the genetic control of $\mathrm{Cd}$ accumulation in leaves was studied using genome-wide association analysis and QTL mapping, whereby candidate genes and favourable alleles were identified [4]. However, studies on the genetic control of $\mathrm{Cd}, \mathrm{As}$, and $\mathrm{Pb}$ in wheat are scarce at best. Here, we found some important MTAs loci for $\mathrm{Cd}$ and $\mathrm{Pb}$ content involving chromosomes $1 \mathrm{~B}, 1 \mathrm{D}, 4 \mathrm{~A}, 4 \mathrm{~B}, 5 \mathrm{~A}, 6 \mathrm{~B}$, and $7 \mathrm{~B}$. This indicates that some important genes need to be studied.

Previous studies have shown that heavy metal ATPases, metal tolerance proteins (MTPs), and natural resistanceassociated macrophage proteins are involved in the deposition of metals in the grain [44]. Plant MTPs are transition metal transporters that catalyse the efflux of $\mathrm{Zn}, \mathrm{Fe}, \mathrm{Mn}, \mathrm{Cd}, \mathrm{Co}$, or $\mathrm{Ni}$ ions from the cytoplasm to the outside of the cell or into subcellular compartments [45, 46]. Therefore, there seems to be a synergy between some heavy metals and some of the beneficial mineral elements or simply, between mineral elements. Once metal ions are absorbed in rice, translocation of $\mathrm{Cd}$ from the roots to the shoots requires loading of $\mathrm{Cd}$ into the xylem from the symplast in the stele, which in turn requires heavy metal ATPase [47]. The Cd-related gene GRMZM2G175576 encoding a heavy metal-transporting ATPase was identified in maize, which is homologous to the rice gene OsHMA3 [4]. However, in our study, six candidate genes associated with $\mathrm{Cd}$ content were predicted in wheat, two of which had multiple effects, that is, $\mathrm{Cd}$ and $\mathrm{Pb}$ content and $\mathrm{Cd}, \mathrm{Fe}$, and $\mathrm{Pb}$ content. Their functions are primarily involved in ion binding, including metal-, Fe-, Ca-, $\mathrm{Cu}-, \mathrm{Mg}-$, and $\mathrm{Zn}$-ion binding. Therefore, there are gene 
interactions among mineral elements, including some that are harmful and some that are beneficial for humans.

\section{Conclusions}

In brief, herein, 17 major MAT loci for nine mineral elements were identified, and 16 candidate genes were predicted. There were some MTA loci clusters found on 12 chromosomes $(1 \mathrm{~A}, 1 \mathrm{~B}, 1 \mathrm{D}, 2 \mathrm{~B}, 3 \mathrm{~B}, 3 \mathrm{D}, 5 \mathrm{~A}, 5 \mathrm{~B}, 6 \mathrm{~B}, 7 \mathrm{~A}$ and 7D). Eight multi-effect MAT loci for controlling more than one trait were detected, mainly distributed on chromosomes 3B, 7B, and 5A. The functions of these candidate genes are primarily involved in ion binding, including metal-, $\mathrm{Fe}-$, $\mathrm{Ca}-$-, $\mathrm{Cu}-, \mathrm{Mg}-$, and $\mathrm{Zn}$-ion binding, ATP binding, ATPase activity, DNA binding, RNA binding, and protein kinase activity. There were gene interactions among some of the mineral elements under study. Therefore, this study provides important loci and gene information for improving mineral element content in the wheat grain. In the future, the candidate genes identified herein should be further studied to elucidate the molecular mechanisms for controlling the content of these mineral elements in the wheat grain.

\section{Abbreviations}

QTL: Quantitative trait loci; GWAS: Genome-wide association study; RILs: Recombinant inbred lines; MQTL: Meta-QTL; MTAs: Marker-trait associations; SNPs: Single nucleotide polymorphisms; DZ: Dezhou; TA: Tai'an; ANOVA: Analysis of variance; MLM: Mixed linear model; NCBI: The National Center for Biotechnology Information; MAPK: Mitogen-activated protein kinase; MTPs: Metal tolerance proteins.
\end{abstract}

\section{Supplementary Information}

The online version contains supplementary material available at https://doi. org/10.1186/s12870-021-03105-3.

Additional file 1: Fig. S1. Standard curves of nine mineral elements in wheat grain. Fig. S2. Manhattan plot of some mineral elements. Table S1. Soil conditions in different planting environments. Table S2. ANOVA analysis of mineral elements. Table S3. All SNP loci significantly associated with beneficial mineral elements $(P<10-4)$. Table S4. All SNP loci significantly associated with heavy metal elements $(P<10-4)$. Table S5. Candidate genes predication and mainly functions of important MATs loci associated with mineral elements.

\section{Acknowledgements}

We thanked Dr. Jirui Wang from Sichuan Agricultural University for analyzing SNP genotyping.

\section{Transparent reporting}

Wheat is a common crop extensively cultivated in the world. This study does not contain any research requiring ethical consent or approval.

\section{Authors' contributions}

Zhiying Deng designed and revised this paper; Wei Wang and Hong Guo analysed the data and wrote the manuscript; Hong Guo, Xiaokang Li, and Chongning Wu investigated and analysed the phenotypic data; Chongning Wu and Hui Yu screened the candidate genes, Guangfeng Chen constructed the map; Jichun Tian reviewed this paper; all authors have read and approved this manuscript.

\section{Funding}

This work is supported by Natural Science Foundation of China (No. 31871613), Shandong Provincial Agriculture Liangzhong Project Foundation of China (No. 2019LZGC01702), Shandong Province Postgraduate Education Tutor Capacity Improvement Program (No. SDYY18114), Natural Science Foundation of Shandong (No. ZR2017LA012) and Shandong "Double Tops" Program. The funding bodies provided financial support to the research project, but were not involved in the design of the study and collection, analysis, and interpretation of data and in writing the manuscript.

\section{Availability of data and materials}

All data used during the current study are included in this published article or are available from the corresponding author on reasonable request from https://pan.baidu.com/s/1X8ISFIrBP0X-u6kMcISGHg. Statement: the database (s) is closed, so before access to this database(s), please request the password from the corresponding author.

\section{Declarations}

Ethics approval and consent to participate

Not applicable.

\section{Consent for publication}

Not applicable.

\section{Competing interests}

The authors declare that they have no competing interests.

\section{Author details}

${ }^{1}$ State Key Laboratory of Crop Biology, Key Laboratory of Crop Biology of Shandong Province, Group of Wheat Quality and Molecular Breeding, College of Agronomy, Shandong Agricultural University, Tai'an, Shandong 271000, P.R. China. ${ }^{2}$ Handan Academy of Agricultural Sciences, Handan, Hebei 056000, P.R. China. ${ }^{3}$ College of Ecology and Garden Architecture, Dezhou University, Dezhou, Shandong 253023, P.R. China.

Received: 12 April 2021 Accepted: 14 June 2021

Published online: 01 July 2021

\section{References}

1. Stein AJ, Qaim M. The human and economic cost of hidden hunger. Food Nutr Bull. 2007;28:125-34.

2. Welch RM, Graham RD. Breeding for micronutrients in staple food crops from a human nutrition perspective. J Exp Bot. 2004;55:353-64.

3. Nawrot T, Plusquin M, Hogervorst J, Roels HA, Celis H, Thijs L, Vangronsveld J, Hecke EV, Staessen JA. Environmental exposure to cadmium and risk of cancer: a prospective population-based study. Lancet Oncol. 2006;7(2):119-26.

4. Zhao XW, Luo LX, Cao YH, Liu YJ, Li YH, Wu WM, Lan YZ, Jiang YW, Gao SB, Zhang ZM, Shen YO, Pan GT, Lin HJ. Genome-wide association analysis and QTL mapping reveal the genetic control of cadmium accumulation in maize leaf. BMC Genomics. 2018;19:91.

5. Navarro-Alarcon M, Lopez-Martınez MC. Essentiality of selenium in the human body: relationship with different diseases. Sci Total Environ. 2000;249(1-3):347-71.

6. Goldhaber SB. Trace element risk assessment: essentiality vs. toxicity. Regul Toxicol Pharmacol. 2003;38(2):232-42.

7. Fraga CG. Relevance, essentiality and toxicity of trace elements in human health. Mol Aspects Med. 2005;26(4-5):235-44.

8. Nielsen $\mathrm{FH}$. The clinical and nutritional importance of chromium-still debated after 50 years of research The nutritional biochemistry of chromium (III). Amsterdam: Elsevier; 2007. p. 265-76.

9. Blust R. Fish physiology. San Diego: Academic; 2011. p. 291-326. 
10. Broadley M, Brown P, Cakmak I. Beneficial elements. In: Ma JF, Rengel Z, Zhao F, editors. Marschner's mineral nutrition of higher plants. 3rd ed. San Diego: Academic; 2012. p. 249-69.

11. Harding KL, Aguayo VM, Webb P. Hidden hunger in South Asia: a review of recent trends and persistent challenges. Publ Health Nutr. 2018;21:785-95.

12. Godecke T, Steiin AJ, Qaim M. The global burden of chronic and hidden hunger: trends and determinants. Glob Food Sec. 2018;17:21-9.

13. Gupta PK, Balyan HS, Sharma S, Kumar R. Biofortifcation and bioavailability of $\mathrm{Zn}$, Fe and Se in wheat: present status and future prospects. Theor Appl Genet. 2021;134:1-35.

14. Crespo-Herrera LA, Velu G, Singh RP. Quantitative trait loci mapping reveals pleiotropic efect for grain iron and zinc concentrations in wheat. Ann Appl Biol. 2016;169:27-35.

15. Crespo-Herrera LA, Govindan V, Stangoulis J. QTL mapping of grain Zn and Fe concentrations in two hexaploid wheat RIL populations with ample transgressive segregation. Front Plant Sci. 2017;8:1800.

16. Yang R, Wang R, Xue W. QTL location and analysis of selenium content in tetraploid wheat grain. Guizhou Agric Sci. 2013;10:1-4.(In Chinese).

17. Pu ZE, Ma YU, He QY. Quantitative trait loci associated with micronutrient concentrations in two recombinant inbred wheat lines. J Integr Agric. 2014;13:2322-9.

18. Wang P, Wang H, Liu Q. QTL mapping of selenium content using a RIL population in wheat. PLoS One. 2017;12:e0184351.

19. Velu G, Singh RP, Crespo-Herrera L. Genetic dissection of grain zinc concentration in spring wheat for mainstreaming biofortifcation in CIMMYT wheat breeding. Sci Rep. 2018;8:13526.

20. Cu ST, Guild G, Nicolson A. Genetic dissection of zinc, iron, copper, manganese and phosphorus in wheat (Triticum aestivum L.) grain and rachis at two developmental stages. Plant Sci. 2020;291:110338.

21. Alomari $\mathrm{DZ}$, Eggert $\mathrm{K}$, von Wirén N. Identifying candidate genes for enhancing grain Zn concentration in wheat. Front Plant Sci. 2018;9:1313.

22. Alomari DZ, Eggert $K$, von Wirén N. Whole-genome association mapping and genomic prediction for iron concentration in wheat grains. Int J Mol Sci. 2019;20:76.

23. Kumar J, Gautam S, Gahlaut V. Genetics of Fe, Zn, $\beta$-carotene, GPC and yield traits in bread wheat (Triticum aestivum $\mathrm{L}$.) using multi-locus and multitraits GWAS. Euphytica. 2018;214:219.

24. Goraf YSA, Ishii T, Kim JS. Genetic variation and association mapping of grain iron and zinc contents in synthetic hexaploid wheat germplasm. Plant Genet Resour. 2016:16:9-17.

25. Bhatta M, Baenziger PS, Waters BM. Genome-wide association study reveals novel genomic regions associated with 10 grain minerals in synthetic hexaploid wheat. Int J Mol Sci. 2018;19:3237.

26. Arora S, Cheema J, Poland J. Genome-wide association mapping of grain micronutrients concentration in Aegilops tauschii. Front Plant Sci. 2019:10:54.

27. Liu Y, Chen YR, Yang Y, Zhang QF, Fu BS, Cai J, Guo W, Shi L, Wu JZ and Chen YH. A thorough screening based on QTLs controlling zinc and copper accumulation in the grain of different wheat genotypes. Environ Sci Pollut. 2020; Research. https://doi.org/10.1007/s11356-020-11690-3.

28. Uauy C, Distelfeld A, Fahima T. A NAC gene regulating senescence improves grain protein, zinc, and iron content in wheat. Science. 2006:314:1298-301.

29. Chen GF, Zhang H, Deng ZY, Wu RG, Li DM, Wang MY, Tian JC. Genomewide association study for kernel weight-related traits using SNPs in a Chinese winter wheat population. Euphytica. 2016;212:173-85.

30. Chen GF, Wu RG, Li DM, Yu HX, Deng ZY, Tian JC. Genome wide association study for seedling emergence and tiller number using SNP markers in an elite winter wheat population. J Genet. 2017;96:177-86.
31. Tiwari VK, Rawat N, Chhuneja P. Mapping of quantitative trait loci for grain iron and zinc concentration in diploid A genome wheat. J Hered. 2009;100:771-6.

32. Tiwari C, Wallwork H, Arun B. Molecular mapping of quantitative trait loci for zinc, iron and protein content in the grains of hexaploid wheat. Euphytica. 2016;207:563-70.

33. Peleg Z, Cakmak I, Ozturk L. Quantitative trait loci conferring grain mineral nutrient concentrations in durum wheat $x$ wild emmer wheat RIL population. Theor Appl Genet. 2009;119:353-69.

34. Balint AF, Röder MS, Hell R. Mapping of QTLs afecting copper tolerance and the $\mathrm{Cu}, \mathrm{Fe}, \mathrm{Mn}$ and $\mathrm{Zn}$ contents in the shoots of wheat seedlings. Biol Plantarum. 2007:51:129-34.

35. Yasmin Z, Paltridge N, Graham R. Measuring genotypic variation in wheat seed iron first requires stringent protocols to minimize soil iron contamination. Crop Sci. 2014;54:255-64.

36. Hao Y, Velu G, Peña RJ. Genetic loci associated with high grain zinc concentration and pleiotropic efect on kernel weight in wheat (Triticum aestivum L.). Mol Breed. 2014;34:1893-902.

37. Velu G, Tutus Y, Gomez-Becerra HF. QTL mapping for grain zinc and iron concentrations and zinc efficiency in a tetraploid and hexaploid wheat mapping populations. Plant Soil. 2017;411:81-99.

38. Liu J, Wu B, Singh RP, Velu G. QTL mapping for micronutrients concentration and yield component traits in a hexaploid wheat mapping population. J Cereal Sci. 2019;88:57-64.

39. Upadhyaya HD, Bajaj D, Das S. Genetic dissection of seed iron and zinc concentrations in chickpea. Sci Rep. 2016;6:24050.

40. Borg S, Brinch-Pedersen H, Tauris B. Wheat ferritins: improving the iron content of the wheat grain. J Cereal Sci. 2012;56:204-13.

41. Tabbita F, Pearce S, Barneix AJ. Breeding for increased grain protein and micronutrient content in wheat: 10 years of the GPC-B1 gene. J Cereal Sci. 2017;73:183-91.

42. Pearce S, Tabbita F, Cantu D. Regulation of Zn and Fe transporters by the GPC1 gene during early wheat monocarpic senescence. BMC Plant Biol. 2014;14:368.

43. Ueno D, Kono I, Yokosho K, Ando T, Yano M, Ma JA. major quantitative trait locus controlling cadmium translocation in rice (Oryza Sativa). New Phytol. 2009;182(3):644-53.

44. Chao DY, Silva A, Baxter I, Huang YS, Nordborg M, Danku J, Lahner B, Yakubova E, Salt DE. Genome-wide association studies identify heavy metal ATPase 3 as the primary determinant of natural variation in leaf cadmium in Arabidopsis Thaliana. PLoS Genet. 2012;8(9):e1002923.

45. Montanini B, Blaudez D, Jeandroz S, Sanders D. Chalot M Phylogenetic and functional analysis of the cation diffusion facilitator (CDF) family: improved signature and prediction of substrate specificity. BMC Genomics. 2007;8:107-22.

46. Tauris B, Borg S, Gregersen PL, Holm PB. A roadmap for zinc trafficking in the developing barley grain based on laser capture microdissection and gene expression profiling. J Exp Bot. 2009;60:1333-47.

47. Uraguchi S, Fujiwara T. Cadmium transport and tolerance in rice: perspectives for reducing grain cadmium accumulation. Rice. 2012;5:5-10.

\section{Publisher's Note}

Springer Nature remains neutral with regard to jurisdictional claims in published maps and institutional affiliations. 